\title{
Composição florística da vegetação de restinga da APA Rio Capivara, Litoral Norte da Bahia, Brasil
}

\author{
Erivaldo Pereira Queiroz ${ }^{1 *}$, Domingos Benício Oliveira Silva Cardoso² \& Marcio Harrison dos Santos \\ Ferreira $^{2}$
}
${ }^{1}$ Herbário Radam-Brasil (HRB), Vigilância Sanitária de Salvador (VISA), Av. Vasco da Gama, 4209, 40285-900, Salvador, Bahia, Brasil.
${ }^{2}$ Programa de Pós-graduação em Botânica, Universidade Estadual de Feira de Santana, Av. Transnordestina s/n, Novo Horizonte, 54036-900, Feira de Santana, Bahia, Brasil.

\begin{abstract}
Resumo - Este trabalho apresenta a lista de plantas vasculares em fragmentos de restinga da Área de Proteção Ambiental Rio Capivara, município de Camaçari, litoral norte da Bahia. O levantamento florístico foi realizado por meio de coletas arbitrárias de indivíduos férteis entre os anos de 2004 e 2006, além de consulta de materiais depositados nos herbários ALCB, HUEFS e HRB. Foi amostrado um total de 358 espécies pertencentes a 343 gêneros e 94 famílias. As famílias com maior número de espécies foram Fabaceae (48 espécies), Rubiaceae (24), Cyperaceae (23), Asteraceae (16), Poaceae (15), Melastomataceae (11), Myrtaceae (11), Orchidaceae (11) e Euphorbiaceae (10). O atual estado de fragmentação e os diversos impactos antrópicos verificados principalmente nos fragmentos de mata de restinga podem ser responsáveis pela baixa riqueza de espécies, quando comparada com outros estudos em vegetação similar. Isso reforça a necessidade urgente de conservação da flora da APA Rio Capivara.
\end{abstract}

Palavras-chave adicionais: conservação, diversidade, endemismo, flora, Mata Atlântica.

\begin{abstract}
Abstact (Floristic composition of remnants of the restinga vegetation in the APA Rio Capivara, northern coast of Bahia State, Brazil) - This paper presents a checklist of the vascular plants from remnants of 'restinga' vegetation of Rio Capivara Environmental Protected Area, in the municipality of Camaçari, Bahia State, Brazil. The survey was based on randomly sampling fertile individuals over the study area from 2004 to 2006, as well as on collections deposited in the herbaria ALCB, HUEFS, and HRB. The list presents a total of 358 species belonging to 343 genera and 94 families. The richest families in number of species were Fabaceae (48 species), Rubiaceae (24), Cyperaceae (23), Asteraceae (16), Poaceae (15), Melastomataceae (11), Myrtaceae (11), Orchidaceae (11), and Euphorbiaceae (10). The current fragmentation and several anthropogenic impacts verified mainly in the restinga forest remnants can be responsible for the low species richness in comparison with other studies in similar vegetation. Our results reinforce an urgent need for conservation of the flora of the APA Rio Capivara.
\end{abstract}

Additional key words: Atlantic Forest, conservation, diversity, endemism, flora.

O termo "restinga" tem sido utilizado para definir as formações vegetais sobre areias holocênicas ou a vegetação continental sobre o areal justamarítimo (Rizzini 1997). As diferentes comunidades vegetais de restinga podem ser encontradas ao longo da costa brasileira em função das condições climáticas e edáficas (Araujo 1992; Silva 2005). Devido às variações de físionomia, estrutura e composição florística, as restingas recebem variadas denominações como: mata de Myrtaceae, mata de restinga, restinga aberta, restinga em moitas, formações de Clusia e mussununga (Araujo \& Henriques 1984).

As restingas ocorrem desde o norte até o sul do Brasil, em áreas fragmentadas sob influência marinha. Segundo Freire (1990), a restinga é um ambiente geologicamente recente e as espécies que a colonizam são principalmente provenientes de outros ecossistemas (e.g., Mata Atlântica e Caatinga), porém com variações fenotípicas devido às condições diferentes do seu ambiente original. Por outro lado, vários trabalhos têm mostrado que as restingas apresentam grande diversidade ecológica (Sugiyama 1998) e características próprias relativas à composição florística e

"Autor para correspondência: eripqueiroz@yahoo.com.br Editor responsável: Pedro Fiaschi

Submetido em: 15 mar. 2010; publicação eletrômica: 11 jun. 2012 estrutura da vegetação (Assumpção \& Nascimento 2000; Pereira et al. 2001; Assis et al. 2004a,b), podendo ainda abrigar uma flora rica em endemismos (Mori et al. 1981; Britto et al. 1993; Silva 2005; Stehmann et al. 2009).

Por estar situada no litoral, onde vive grande parte da população brasileira, a vegetação de restinga está entre os ambientes mais ameaçados do Domínio da Mata Atlântica (Stehmann et al. 2009). Embora muitas áreas com vegetação de restinga estejam protegidas por unidades de conservação (MMA 2000), a ampliação da malha rodoviária no Brasil e a especulação imobiliária associadas ao crescente fluxo turístico vêm causando sérios danos à vegetação nativa. Essa antropização crescente pode ser responsável também pela falta de refúgio para várias espécies de lagartos que são adaptadas somente a esses tipos de ambiente (Teixeira 2001), e pelas alterações nas comunidades de abelhas que participam de processos ecológicos importantes (Costa \& Ramalho 2001). A relevância dos estudos descritivos sobre vegetação de restinga para a preservação ambiental e manejo de unidades de conservação reside no fornecimento de dados, por exemplo, para estudos sobre a dinâmica de comunidades. Essas pesquisas procuram, além disso, facilitar a escolha de espécies cujas populações mereçam estudos mais 


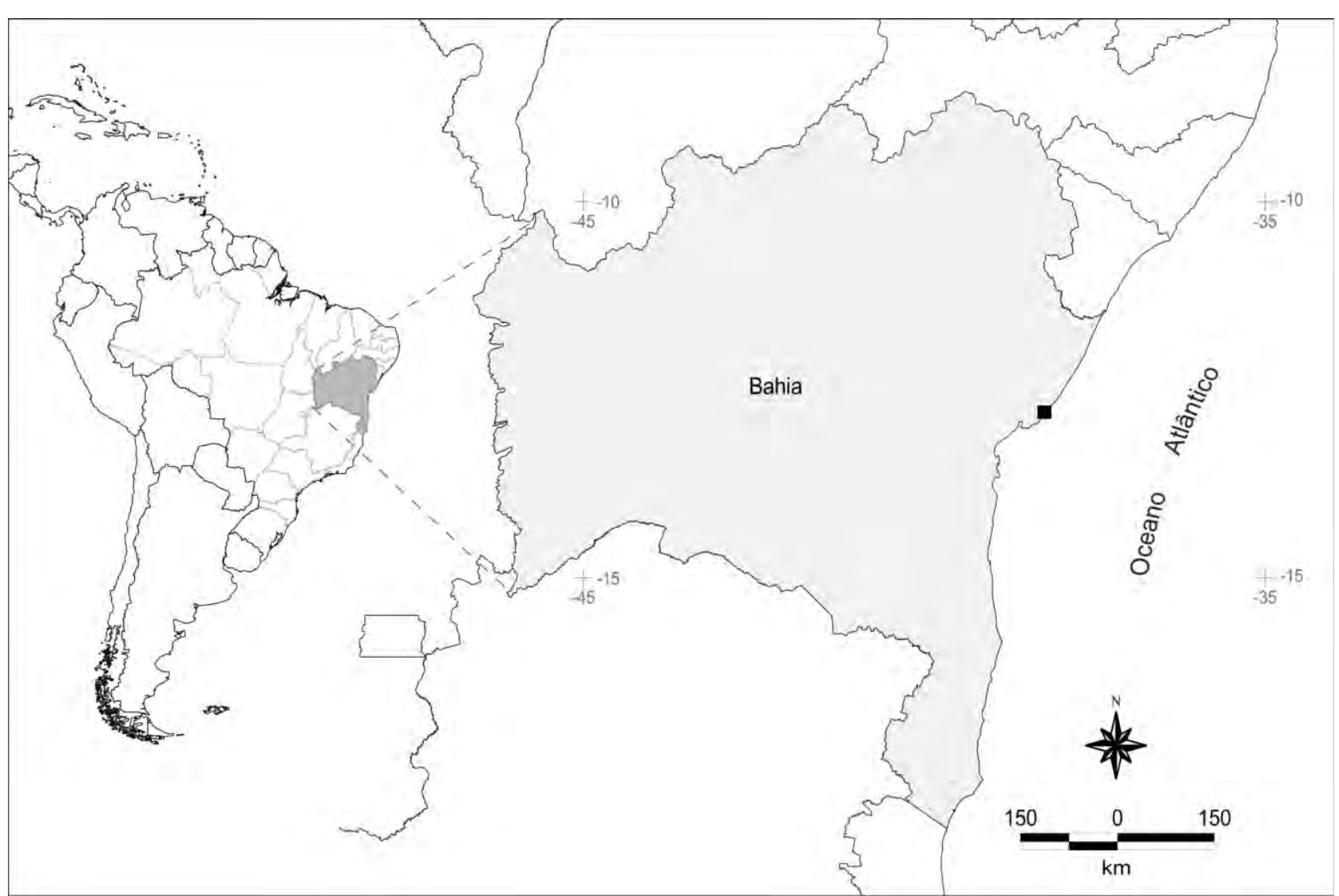

Figura 1. Localização da área de estudo em Arembepe, município de Camaçari, litoral norte da Bahia, Brasil.

detalhados, objetivando a preservação de espécies raras (Pereira et al. 2001).

Até o momento, a maior parte dos estudos no Brasil relacionados à caracterização florística ou estrutural da vegetação de restinga tem sido realizada nas Regiões Sul e Sudeste do país (e.g., Araujo \& Henriques 1984; César \& Monteiro 1995; Sugiyama 1998; Assumpção \& Nascimento 2000; Pereira \& Araujo 2000; Pereira \& Assis 2000; Müller \& Waechter 2001; Pereira et al. 2001; Assis et al. 2004a,b). Mesmo apresentando o mais extenso litoral do Brasil, com aproximadamente $1.500 \mathrm{~km}$, poucos trabalhos foram realizados sobre a composição florística em áreas de restinga no estado da Bahia. Alguns deles abordam em um contexto mais amplo a flora do litoral norte, que incluí também o município de Camaçari (Pinto et al. 1984a,b; IBGE 2004; Queiroz 2007). Outros dois foram desenvolvidos na APA (Área de Proteção Ambiental) da Lagoa do Abaeté (Britto \& Noblick 1984; Britto et al. 1993) e um outro nas ilhas oceânicas do Parque Nacional Marinho de Abrolhos (Kemenes 2003). Além destes, um estudo florístico bem detalhado vem sendo desenvolvido ao longo dos últimos quatro anos na região de Entre Rios, também no litoral Norte (A. Popovkin, com. pess.), e que tem resultado na descoberta de diversas espécies novas, além de um amplo conhecimento da distribuição de espécies antes conhecidas apenas para o sul da Bahia.

A escassez de informações florísticas sobre a vegetação de restinga da Bahia implica ainda em uma grande lacuna de conhecimento, que poderia ser útil em comparações fitogeográficas com outras restingas do Brasil. Neste sentido, o presente estudo foi delineado tendo como objetivo principal a realização do levantamento das plantas vasculares em diferentes fragmentos de restinga da APA Rio Capivara, município de Camaçari, visando contribuir para o conhecimento desse tipo vegetacional na Bahia, e subsidiar futuros estudos fitogeográficos.

\section{Material e Métodos}

Os fragmentos de vegetação de restinga estudados localizam-se em Arembepe, município de Camaçari (Figuras 1 e 2), entre as coordenadas aproximadas $12^{\circ} 44^{\prime} 30^{\prime \prime}-12^{\circ} 46^{\prime} 26^{\prime \prime} \mathrm{S}$ e $38^{\circ} 09^{\prime} 30^{\prime \prime}-38^{\circ} 11^{\prime} 05^{\prime \prime} \mathrm{W}$, e são reconhecidos como parte da Área de Proteção Ambiental Rio Capivara. Esses fragmentos formam um mosaico junto a áreas úmidas (brejos e lagoas) associadas aos cordões de dunas, fragmentos de Mata Atlântica, rios, manguezais e praias (SEMARH/DUC \& CRA 2005). Os trechos de restinga arbórea amostrados possuem um mínimo de 100 $\mathrm{m}^{2}$, e às vezes distam $300 \mathrm{a} 600 \mathrm{~m}$ do fragmento mais próximo ou de uma área de restinga arbórea mais contínua, com cerca de $1.500 \mathrm{~m}^{2}$. No total, a área de estudo abrangeu cerca de 90 ha. A tipologia climática da região é do tipo úmido a sub-úmido, com temperatura média de 19,4 a $24,6^{\circ} \mathrm{C}$ e precipitação anual média de 1.100 a $2.000 \mathrm{~mm}$, com chuvas concentradas no outono-inverno (SEI 1998).

Foi analisado o tipo de solo da área de estudo a partir de 

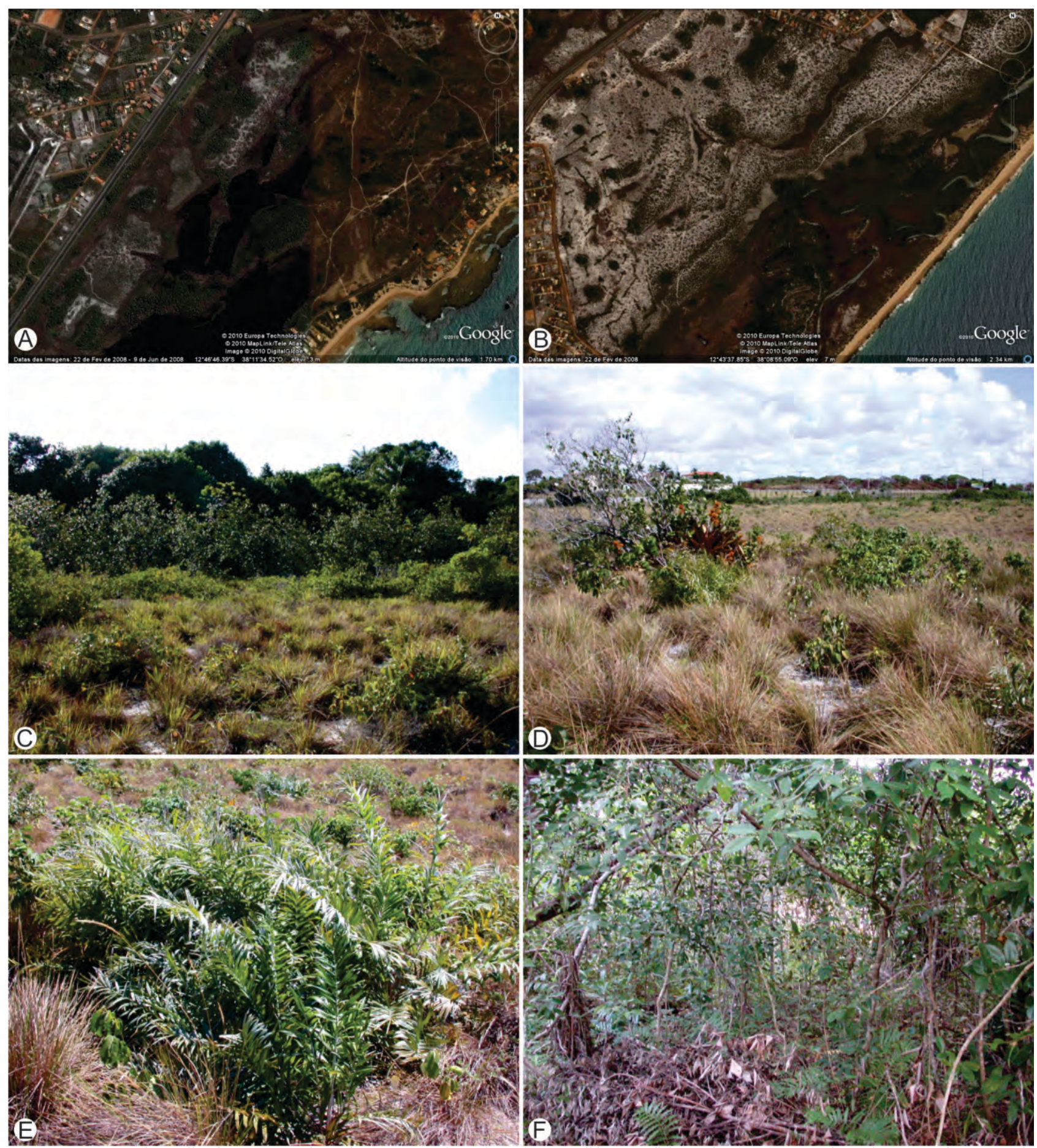

Figura 2. Imagens de satélite da vegetação de restinga em Arembepe (A e B) e suas diferentes fisionomias (C-F): C- restinga aberta com fragmento de restinga arbórea ao fundo; D-E- restinga aberta; F-interior da restinga arbórea (fotos: A, B- Google Earth; C-F-Synara M. Leal).

60 amostras com aproximadamente $0,5 \mathrm{~kg}$, a $20 \mathrm{~cm}$ de profundidade por $10 \mathrm{~cm}$ de diâmetro. As amostras foram coletadas em três pontos $(0,50$ e $100 \mathrm{~m})$ em cada um dos 20 transectos de $100 \mathrm{~m}$ de comprimento, de forma a representar toda área de acordo com as variações da vegetação e do solo. As amostras foram analisadas pelo laboratório de solos da Empresa Baiana de Desenvolvimento Agropecuário (EBDA).

O levantamento florístico foi realizado por meio de coletas arbitrárias nos diferentes fragmentos de vegetação de restinga. Foram visitados 16 fragmentos, compostos pelas seguintes fitofisionomias: nove fragmentos de restinga arbórea, vegetação formada por um estrato arbóreo e um sub-bosque rico em arbustos e ervas; quatro fragmentos formados por restinga aberta, vegetação predominatemente herbácea, com arbustos esparsos e onde há um maior depósito de areias quartzosas; e três áreas compostas por vegetação periodicamente alagada. Entre os 
anos de 2004 e 2006, foram feitas 25 coletas com duração de até 5 dias cada, sendo que foi possível amostrar as espécies em diferentes períodos chuvosos e secos. Além disso, foram realizados levantamentos de materiais já depositados nos herbários ALCB, HRB e HUEFS (acrônimos segundo Holmgren et al. 1990). O material botânico coletado durante as excursões foi incorporado tanto na coleção do HRB quanto na do HUEFS, sendo que as duplicatas foram enviadas para especialistas.

A identificação do material foi realizada com o auxílio de bibliografia especializada, por comparação com o material depositado nos herbários HRB e HUEFS e através da consulta a especialistas nos diferentes grupos. Neste trabalho, foi adotado o sistema APG III (2009) para classificação das famílias de angiospermas e o sistema de Smith et al. (2006) para as monilófitas.

\section{Resultados}

Lista de espécies. A flora vascular da vegetação de restinga da APA Rio Capivara foi representada por 358 espécies distribuídas em 343 gêneros e 94 famílias (Apêndice; para imagens de representantes da flora, veja
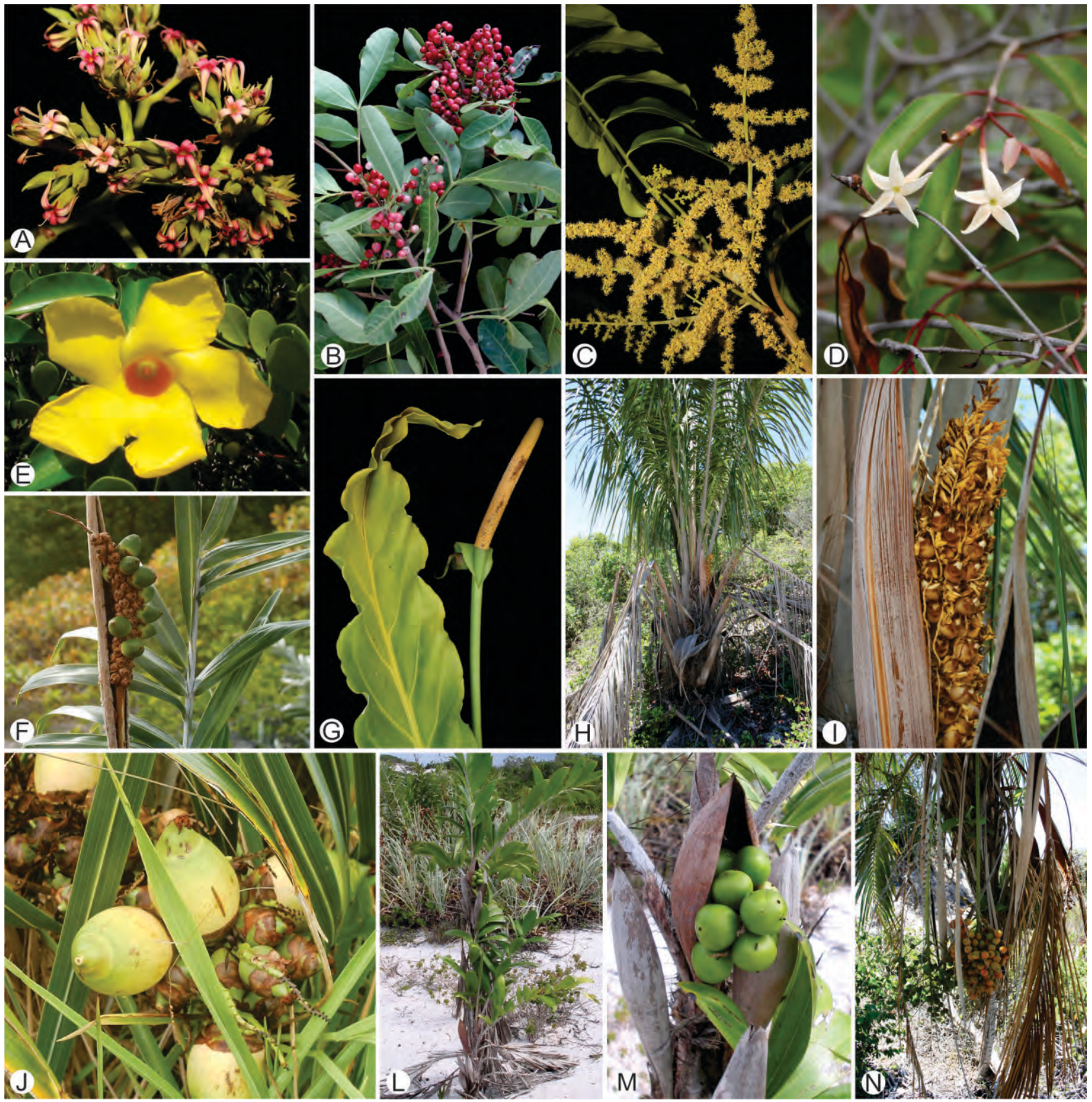

Figura 3. Representantes da flora vascular da restinga da APA Rio Capivara, Bahia, Brasil: A-Anacardium occidentale; B- Schinus terebinthifolius; CTapirira guianensis; D-Hancornia speciosa; E-Mandevilla scabra; F-Allagoptera brevicalyx; G-Anthurium affine; H-J-Attalea funifera; L-M-Bactris soeiroana; N-Syagrus schizophylla (fotos: Erivaldo P. Queiroz). 

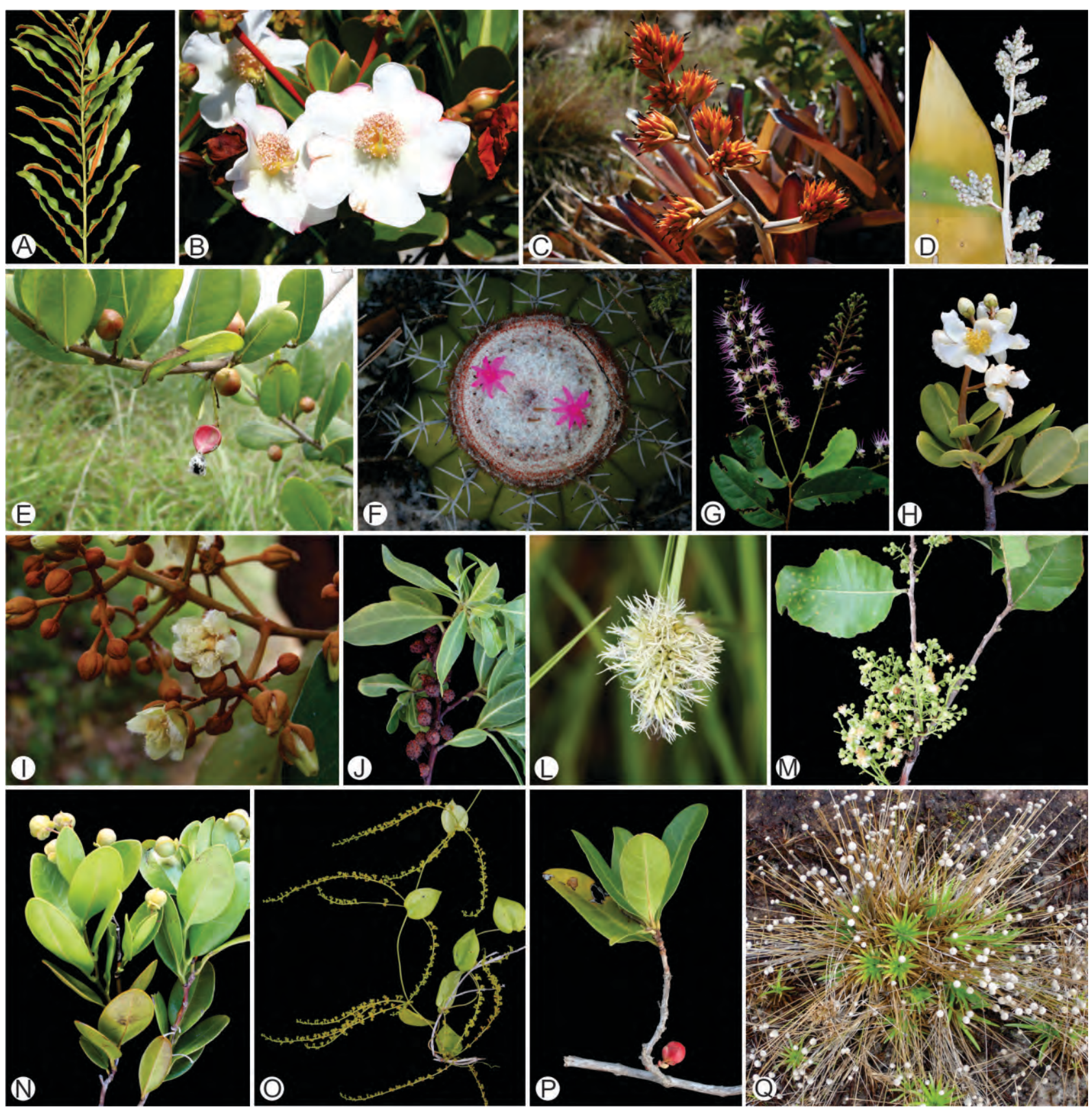

Figura 4. Representantes da flora vascular da restinga da APA Rio Capivara, Bahia, Brasil: A- Blechnum serrulatum; B- Bonnetia stricta; C- Aechmea marauensis; D- Hohenbergia littoralis; E- Protium bahianum; F- Melocactus violaceus subsp. margaritaceus; G- Hirtella racemosa; H- Kielmeyera argentea; I- Vismia guianensis; J- Conocarpus erectus; L-Rhynchospora cephalotes; M- Curatella americana; $\mathbf{N}$-Davilla flexuosa; $\mathbf{O}-$ Dioscorea ovata; P-Doliocarpus sellowianus; Q- Comanthera imbricata (fotos: Erivaldo P. Queiroz).

Figuras 3-8). As angiospermas representaram 352 espécies, pertencentes a 236 gêneros e 88 famílias, enquanto as monilófitas foram representadas por apenas sete espécies, em sete gêneros e seis famílias. As famílias de angiospermas mais bem representadas em número de espécies foram Fabaceae (48 espécies), Rubiaceae (24), Cyperaceae (23), Asteraceae (16), Poaceae (15), Melastomataceae (11), Myrtaceae (11), Orchidaceae (11) e Euphorbiaceae (10), que juntas somaram quase $50 \%$ do total da flora amostrada. As demais famílias foram compostas por 189 espécies, sendo que 42 delas foram representadas por apenas uma espécie. Os gêneros mais diversos foram Cyperus (8 espécies), Polygala (6), Byrsonima (5), Chamaecrista (5), Myrcia (5), Paspalum (5) e Rhynchospora (5). Na área estudada, houve uma predominância de espécies herbáceas, sendo a distribuição dos hábitos assim representada: ervas (142 espécies), arbustos (95), subarbustos (57), árvores (24), trepadeiras (34), hemi e holoparasitas (5) e epífitas (1).

Caracterização do solo. O solo da área de estudo 

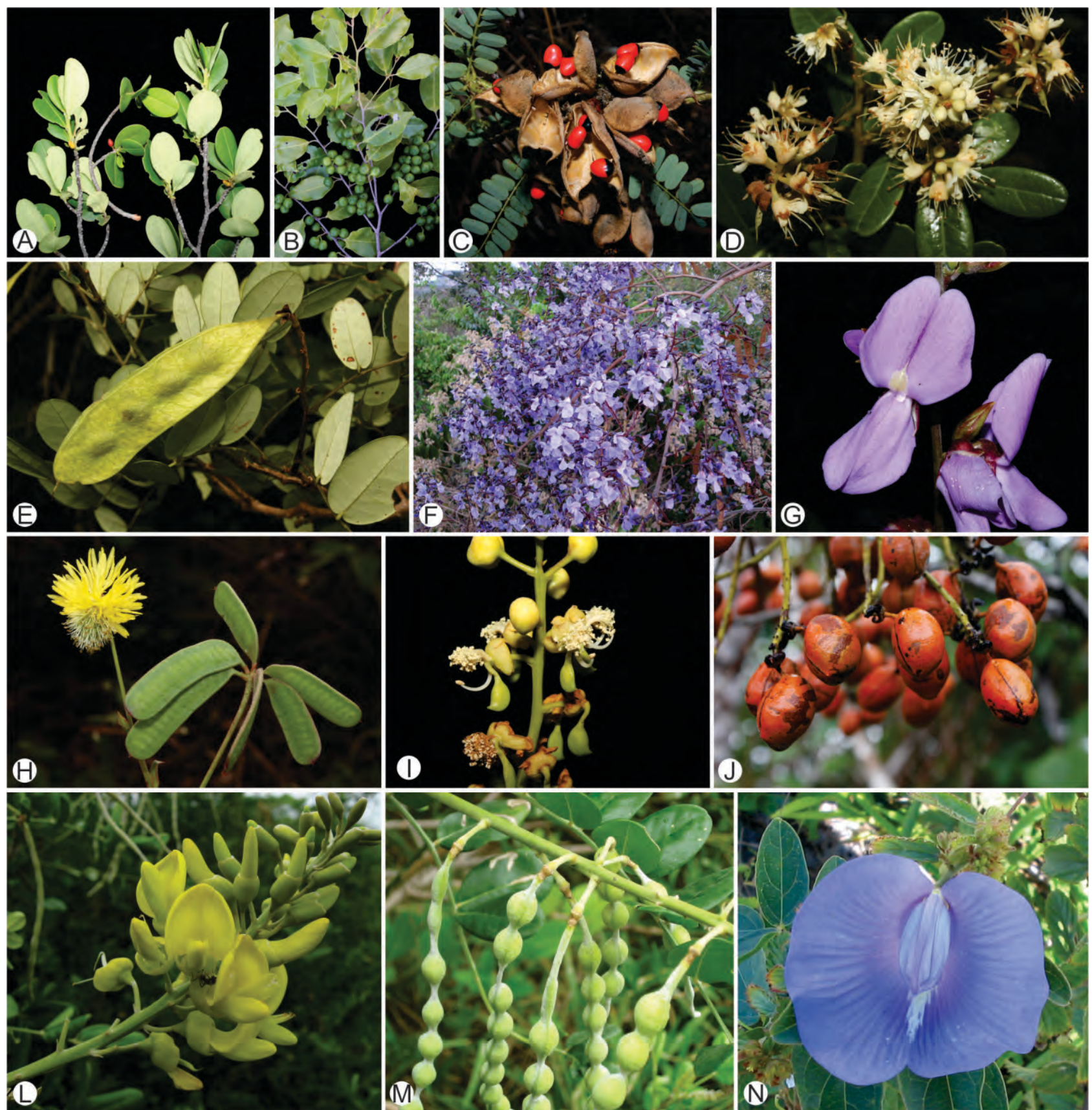

Figura 5. Representantes da flora vascular da restinga da APA Rio Capivara, Bahia, Brasil: A- Erythroxylum leal-costae; B- Pera glabrata; C- Abrus precatorius; D-E- Leptolobium bijugum; F- Bowdichia virgilioides; G- Dioclea lasiophylla; H- Neptunia plena; I-J- Swartzia apétala; L-M- Sophora tomentosa; N-Centrosema brasilianum (fotos: A, B e N-Erivaldo P. Queiroz; C-M- Domingos B.O.S. Cardoso).

caracteriza-se como Neossolos Quartzarênicos do Período Quaternário, formados pela deposição flúvio-lacustre, eólica e, especialmente, marinha. A estrutura é do tipo grão simples com pH variando entre 4,8 e 6,5 (média 5,6), sendo caracterizado, portanto, como pouco ácido. Além disso, o solo é sobretudo pobre em matéria orgânica, variando entre 0,3 a 3,0\%, com $8,71 \mathrm{~g} / \mathrm{kg}$ em média, porém nas áreas encharcadas a matéria orgânica pode chegar a 19,4\%. Na restinga arbórea, o solo também apresenta maior quantidade de matéria orgânica, provavelmente devido à serrapilheira, a qual, juntamente com a maior umidade local, promove maior desenvolvimento e acúmulo de matéria orgânica, favorecendo a colonização do ambiente por algumas espécies (e.g., Coccoloba alnifolia, Guettarda platypoda, Maytenus distichophylla, Ocotea notata e Protium bahianun). Com relação à granulometria, a composição do substrato apresentou as seguintes proporções: areia fina $65 \%$, areia grossa $32,2 \%$, argila $2,7 \%$ e silte $0,1 \%$.

Caracterização da vegetação. Nos fragmentos de restinga arbórea estudados em Arembepe, observa-se um dossel contínuo entre 7 e $8 \mathrm{~m}$, caracterizado principalmente pelas espécies Bowdichia virgilioides, Emmotum affine, Inga capitata e Ocotea notata. Entretanto, nos fragmentos 

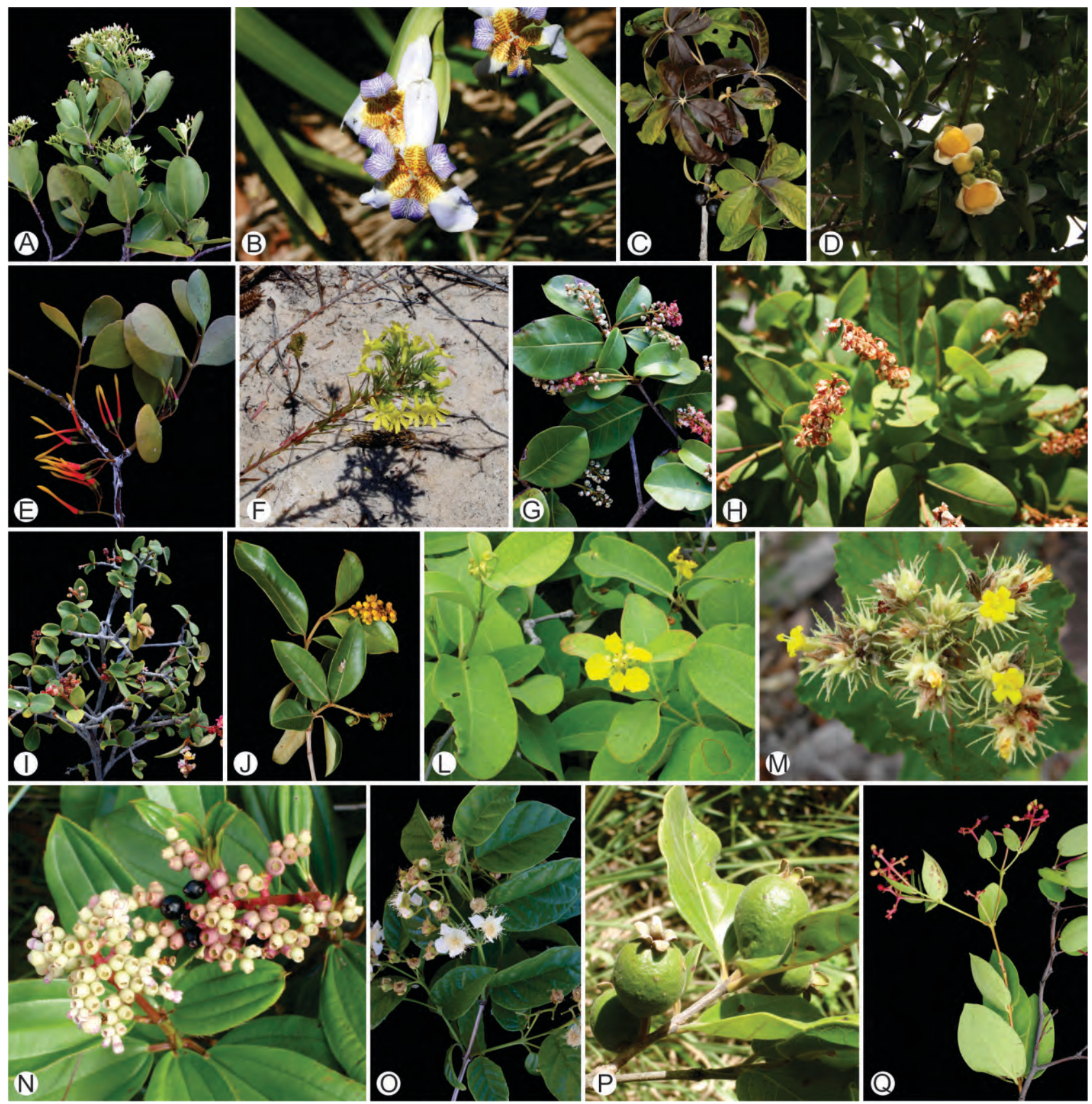

Figura 6. Representantes da flora vascular da restinga da APA Rio Capivara, Bahia, Brasil: A- Humiria balsamifera; B- Neomarica sabinii; C- Vitex cymosa; D- Eschweilera ovata; E- Psittacanthus dichroos; F- Cuphea flava; G- Byrsonima bahiana; H- Byrsonima coccolobifolia; I- Byrsonima microphylla; J- Byrsonima sericea; $\mathbf{L}$-Stigmaphyllon paralias; M- Waltheria cinerascens; $\mathbf{N}$ - Miconia ciliata $; \mathbf{O}$ - Campomanesia dichotoma; $\mathbf{P}-$ Psidium guineense- Q- Guapira pernambucensis (fotos: A-Le N-P- Erivaldo P. Queiroz; M- Domingos B.O.S. Cardoso).

do cordão interno de dunas, Vochysia lucida parece ser a espécie mais frequente, às vezes formando o dossel. O subbosque é formado por muitas espécies arbustivas, tais como Byrsonima microphylla, Croton sellowii, além de várias espécies de Myrtaceae. Neste estrato, é possível encontrar também ervas pertencentes às famílias Rubiaceae, Poaceae e Bromeliaceae, além de espécies epifíticas, como Brassavola tuberculata e a hemiparasita Psittacanthus robustus, que ocorre frequentemente sobre Vochysia lucida.

As áreas periodicamente alagadas são caracterizadas principalmente pela presença de Kielmeyera argentea,
Leiothrix sp., Bacopa gratioloides, Hydrolea spinosa, Neptunia plena, Xyris laxifolia, Pterolepis sp., Koellensteinia abaetana, Sauvagesia erecta, Epistephium lucidum e espécies saprofíticas de Utricularia.

$\mathrm{Na}$ restinga aberta, é comum a presença de Canavalia rosea, Dalbergia ecastaphyllum e Chrysobalanus icaco em locais mais próximos da praia. Nos locais entre os fragmentos de restinga arbórea, a vegetação é composta principalmente por espécies herbáceas, como Hohenbergia littoralis, Melocactus violaceus subsp. margaritaceus, Marcetia ericoides, Actinocephalus ramosus, Vellozia 

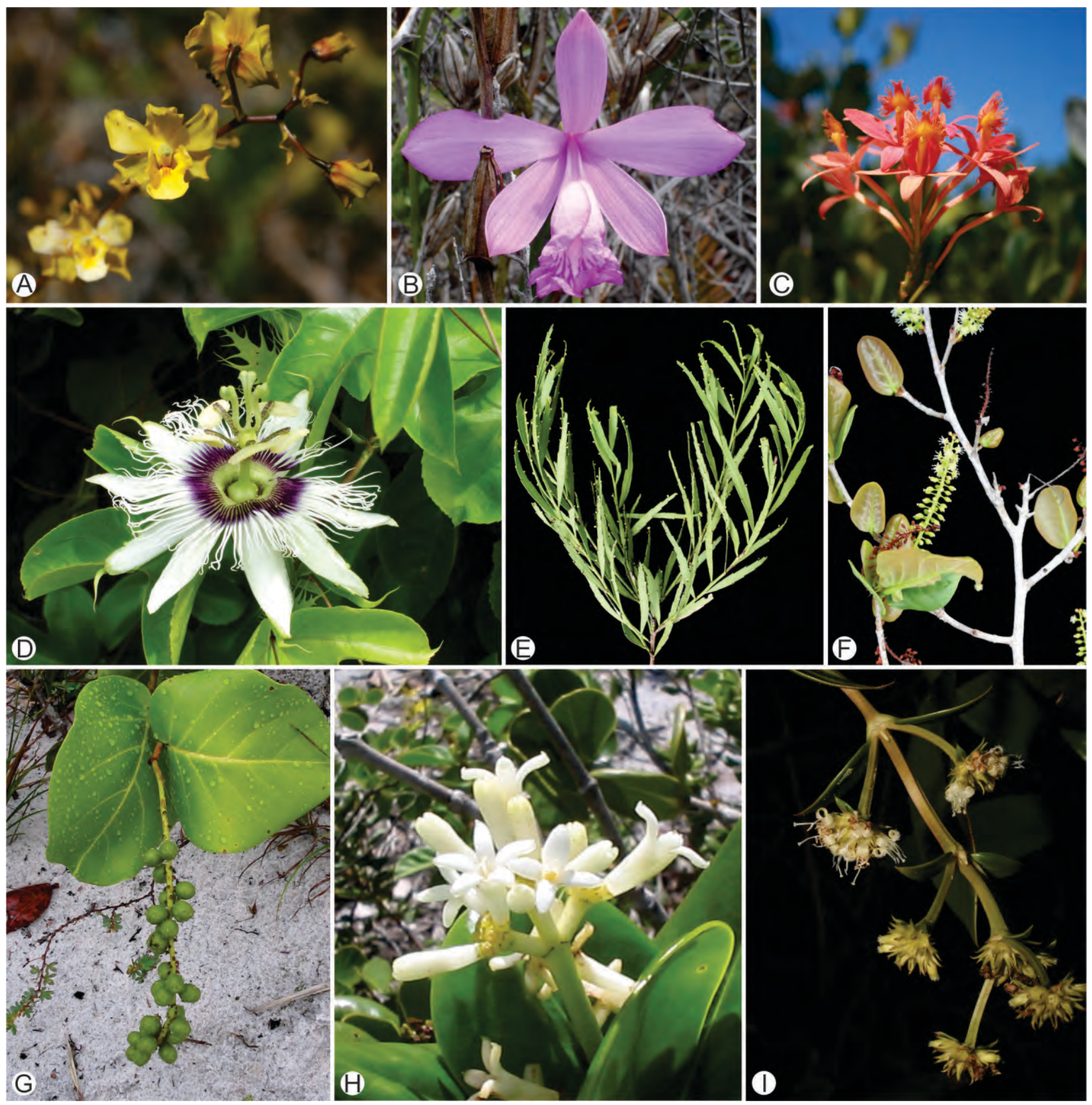

Figura 7. Representantes da flora vascular da restinga da APA Rio Capivara, Bahia, Brasil: A- Cyrtopodium holstii; B- Epistephium lucidum; CEpidendrum cinnabarinum; D- Passiflora edulis; E- Phyllanthus klotzschianus; F- Coccoloba ramosissima; G- Coccoloba laevis; H- Chiococca brachiata; I-Denscantia monodon (fotos: A-H-Erivaldo P. Queiroz; I- Domingos B.O.S. Cardoso).

dasypus, Cyrtopodium holstii e Epidendrum cinnabarinum, além de várias espécies de Poaceae. Nesta fitofisionomia, os arbustos são distribuídos esparsamente e formando moitas, sendo representados principalmente por Allagoptera brevicalyx, Ouratea suaveolens, Esenbeckia grandiflora subsp. brevipetiolata, Swartzia apetala, Manilkara decrescens, Guettarda angelica e Protium bahianum.

O processo de fragmentação e degradação de algumas áreas de restinga da APA Rio Capivara também foi verificado nas três fitofisionomias estudadas. Nos fragmentos onde o processo de antropização vem sendo intensificado, destaca-se a presença de espécies típicas de ambiente de borda, tais como Annona glabra, Byrsonima sericea, Cecropia pachystachya, Curatella americana, Myrcia guianensis, Pera glabrata e Pavonia cancellata.

\section{Discussão}

Diversidade de espécies. As comunidades arbustivas de restinga têm sido classificadas, a partir de suas características fisionômicas, em formações abertas ou descontínuas (de Clusia, de Ericaceae, de Arecaceae) e 

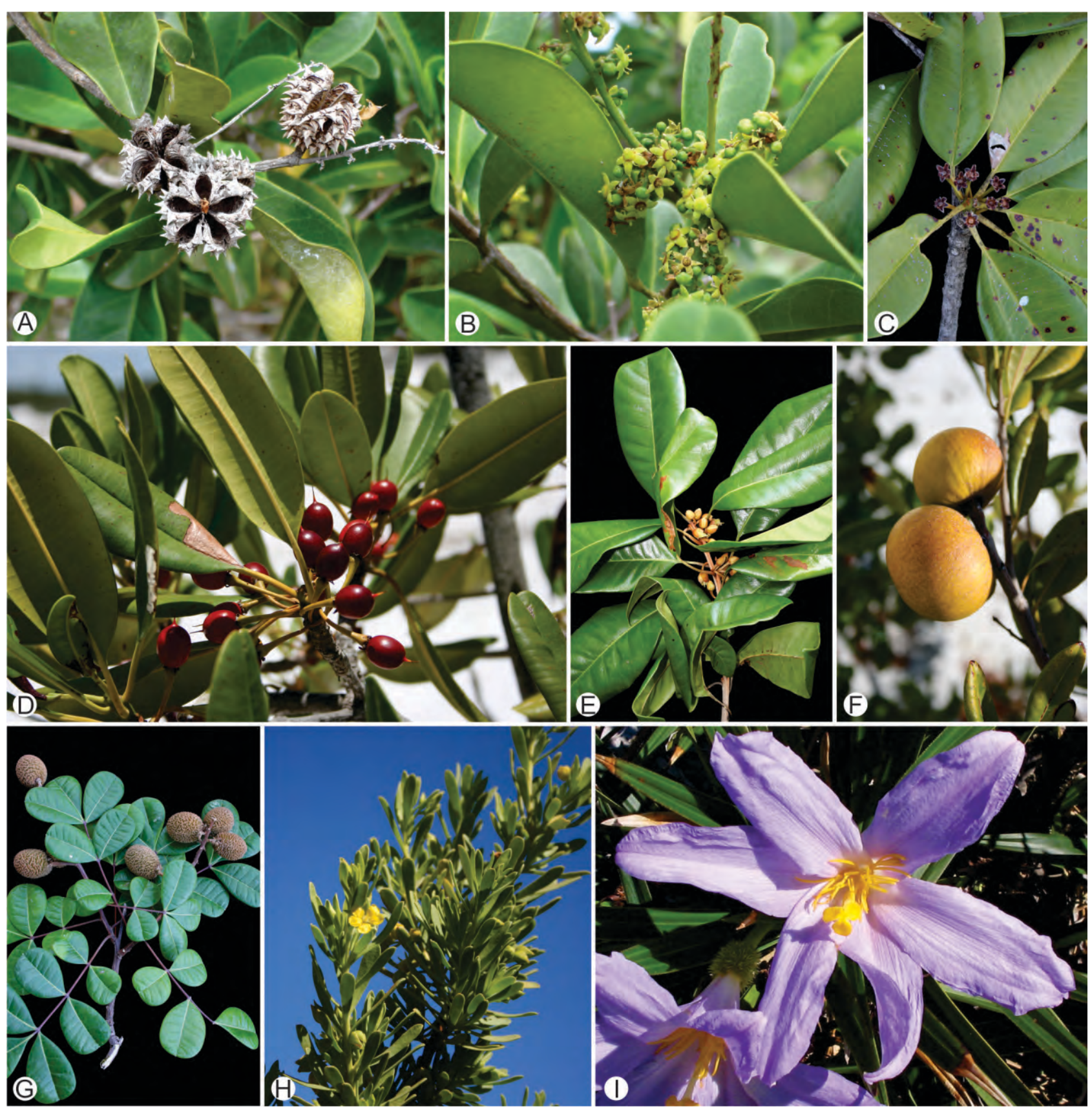

Figura 8. Representantes da flora vascular da restinga da APA Rio Capivara, Bahia, Brasil: A-B- Esenbeckia grandiflora subsp. brevipetiolata; C-DManilkara salzmannii; E-F-Pouteria grandiflora; G-Simaba cuneata; H-Suriana maritima; I- Vellozia dasypus (fotos: Erivaldo P. Queiroz).

fechadas ou contínuas (de Myrtaceae e pós-praia) (Araujo \& Henriques 1984; Araujo 1992; Assumpção \& Nascimento 2000; Silva 2005). Assumpção \& Nascimento (2000) caracterizaram a formação mata de restinga como uma formação de dossel contínuo, com porte de até $6 \mathrm{~m}$ de altura e elevado número de espécies de Myrtaceae, além de outros atributos estruturais. Os fragmentos de restinga arbórea da APA Rio Capivara parecem se enquadrar nessa descrição, enquanto as demais áreas poderiam ser chamadas de formações abertas de Allagoptera (Arecaceae). No entanto, é importante ressaltar que o estabelecimento de uma nomenclatura para as diferentes comunidades de restinga, principalmente em relação àquelas que ocorrem ao longo do litoral da Bahia, poderia ser ainda melhor investigado por meio de uma perspectiva biogeográfica. Neste sentido, uma análise fitogeográfica utilizando os diferentes estudos já desenvolvidos na costa atlântica brasileira pode ser importante na tentativa de elaborar uma classificação de vegetação mais abrangente para as restingas.

Nos levantamentos florísticos realizados no litoral norte da Bahia pelo Projeto RADAM-BRASIL, segundo o IBGE (2004), são listadas apenas 36 espécies para a vegetação de restinga de Arembepe. Portanto, o presente estudo amplia consideravelmente o conhecimento acerca da diversidade florística na área. 
Como observado também em outros estudos sobre vegetação de restinga (e.g., Mantovani 1992; Assumpção \& Nascimento 2000; Lemos et al. 2001; Pereira et al. 2001; Assis et al. 2004b; Scherer et al. 2005), Myrtaceae apresentou o maior número de espécies entre as famílias mais bem representadas na restinga da APA Rio Capivara. Num estudo em floresta de restinga em Guarapari (Espírito Santo), Assis et al. (2004b) verificaram que Myrtaceae, Orchidaceae, Bromeliaceae e Rubiaceae foram as famílias que apresentaram maior riqueza de espécies. Dado que essas mesmas famílias foram também mencionadas como as mais ricas em diversos trabalhos ao longo da costa brasileira, elas foram apontadas por Assis et al. (2004b) como as principais famílias das restingas brasileiras. No presente estudo, constatou-se que três destas, Rubiaceae, Orchidaceae e Myrtaceae, figuraram entre aquelas com maior riqueza de espécies.

Diferente do que foi observado por Assis et al. (2004b) e Fabris \& César (1996), Fabaceae foi a família com maior número de espécies neste trabalho, nas dunas do Abaeté, em Salvador (Britto et al. 1993), em outras áreas de restinga do litoral norte da Bahia (IBGE 2004) e em diversos outros estudos realizados na costa brasileira (Silva \& Oliveira 1989; Pereira \& Araujo 2000; Pereira \& Assis 2000).

Uma comparação da diversidade florística da APA Rio Capivara com a de outras áreas de restinga do Brasil mostra que a sua vegetação compartilha poucas espécies com as restingas da Região Sudeste (ver lista de espécies em Mantovani 1992; Assumpção \& Nascimento 2000; Pereira \& Assis 2000; Pereira et al. 2001, 2004; Assis et al. 2004b). Nessas áreas são comuns várias espécies de distribuição ampla nas restingas do Brasil, tais como Chrysobalanus icaco e Dalbergia ecastaphyllum, que ocorrem desde o Pará até São Paulo (Araujo \& Henriques 1984). Esse padrão de distribuição contínuo na planície litorânea brasileira também caracteriza Coccoloba ramosissima (Melo 1996). A similaridade florística da área de estudo parece ser maior com a vegetação das dunas do Abaeté (Britto et al. 1993; Queiroz, dados não publicados), como revelado pelo grande número de espécies compartilhadas (205 espécies), a exemplo de Byrsonima microphylla, Comolia ovalifolia, Cuphea brachiata, Davilla flexuosa, Encyclia dichroma, Hohenbergia littoralis, Kielmeyera argentea, Koellensteinia abaetana, Krameria bahiana, Protium bahianum e Syagrus schizophylla.

Endemismo, fragmentação e implicações para conservação. A lacuna nos estudos de vegetação de restinga no estado da Bahia tem prejudicado a implantação de medidas necessárias à conservação desse ecossistema, como a escolha de áreas de preservação permanente adicionais. A necessidade de implantação de medidas efetivas de preservação das restingas ao longo do litoral baiano pode ser evidenciada pelo seu elevado número de espécies endêmicas. Na região do litoral norte do estado e em Salvador, existem registros de pelo menos 19 espécies endêmicas: Allagoptera brevicalyx, Bactris soeiroana (Arecaceae), Blepharodon costae (Apocynaceae), Byrsonyma microphylla (Malpighiaceae), Calycolpus legrandii (Myrtaceae), Chamaecrista salvatoris
(Fabaceae-Caesalpinioideae), Duguetia moricandiana (Annonaceae), Eriope blanchetii (Lamiaceae), Erythroxylum leal-costae (Erythroxylaceae), Hohenbergia littoralis (Bromeliaceae), Kielmeyera argentea (Clusiaceae), Koellensteinia abaetana (Orchidaceae), Mimosa carvalhoi (Fabaceae-Mimosoideae), Moldenhawera nutans (Fabaceae-Caesalpinioideae), Ouratea rotundifolia (Ochnaceae), Poecilanthe itapuana (Fabaceae-Faboideae), Protium bahianum (Burseraceae), Tetracera boomii (Dilleniaceae) e Vanilla bahiana (Orchidaceae) (Britto et al. 1993).

Além disso, em alguns pontos do litoral norte também é encontrada Brodriguesia santosii R.S.Cowan (E.P. Queiroz, obs. pess.), espécie única de um gênero de Fabaceae-Caesalpinioideae endêmico da Bahia e que era conhecido somente na Floresta Atlântica do sul do Estado (Thomas et al. 2003). Nas restingas da região de Mata de São João, também no litoral norte, foi coletada uma espécie ainda não descrita de Swartzia (Fabaceae-Faboideae) (V.F. Mansano, com. pess.). Já em Entre Rios, numa região próxima de Mata de São João, um levantamento florístico em andamento já possui pelo menos 380 espécies de angiospermas catalogadas, dentre as quais incluem-se espécies novas de Bauhinia (Fabaceae) e Lecythis (Lecythidaceae), além das espécies recentemente descritas Davilla sessilifolia (Dilleniaceae), Mezilaurus revolutifolia (Lauraceae) e Spigelia genuflexa (Loganiaceae) (Fraga 2008; Alves et al. 2011; Popovkin et al. 2011).

Tendo em vista os padrões de fragmentação e distúrbios observados na área estudada na APA Rio Capivara, e por meio de informações obtidas com moradores locais, podese inferir que o processo de descaracterização ambiental, o extrativismo predatório para o uso paisagístico (e.g., Encyclia dichroma) e a obtenção de lenha continuam ocorrendo. Por exemplo, o extrativismo de espécies de epífitas (e.g., Brassavola tuberculata) gera um impacto adicional na vegetação de restinga da APA Rio Capivara, dado que essas espécies são retiradas presas aos suportes, prejudicando também o forófito.

Outro aspecto agravante na APA Rio Capivara são os efeitos da fragmentação no estabelecimento das comunidades vegetais e na colonização desses ambientes diante da crescente alteração da vegetação e extração ilegal de espécies. Vale ressaltar também que a fragmentação causa alterações em alguns processos ecológicos importantes, tais como a polinização e a dispersão de sementes (Meffe et al. 1997; Lundberg \& Ingvarsson 1998; Lennartsson 2002). Além disso, algumas condições de borda relacionadas ao microclima (e.g., luz e regime de vento) e alterações no aspecto vegetacional têm sido consideradas como responsáveis pelo aumento da riqueza de espécies adaptadas a ambientes alterados (Freidenburg 1997; Meffe et al. 1997), contribuindo para a perda da diversidade florística.

Levantamentos etnoecológicos na área podem ser uma ferramenta importante para o resgate e a valorização do conhecimento local, assim como para a identificação de outros impactos em alguns processos ecológicos. O conhecimento tradicional já foi destacado como importante 
para a conservação em vários outros ecossistemas (Posey 1983; Marques 1995; Begossi 1998; Albuquerque 1999a,b; Diegues 2000; Albuquerque \& Andrade 2002). No entanto, ainda há uma escassez de estudos dessa natureza em ambientes de restinga no Brasil (e.g., Fonseca-Kruel \& Peixoto 2004; Melo et al. 2008; Miranda \& Hanazaki 2008).

Além dos vários impactos ambientais verificados nas matas de restinga, outros ainda têm sido observados nas áreas periodicamente alagadas pelo rio Capivara, como a deposição de lixo e esgotos a céu aberto, queimadas, aterros, ocupação desordenada e a implantação de condomínios. As áreas alagáveis e ecossistemas associados exercem importantes funções na ecologia da paisagem (Junk 2002; Kita \& Souza 2003) e contribuem consideravelmente para o bem-estar de grande parte da população humana, mas encontram-se seriamente ameaçadas porque ainda são, em geral, consideradas recursos livres de terras e águas (Junk 2002). Este mesmo autor afirma ainda que muitos países tropicais não têm poder econômico, científico e capacitação tecnológica e/ou infraestrutura administrativa para reagir adequadamente aos desafios do crescente aumento populacional, às pressões sobre as áreas litorâneas, e à economia global com relação à utilização sustentável dos recursos.

Políticas ambientais "equivocadas" ou inadequadas são um dos fortes entraves ao correto planejamento e conservação nesses ecossistemas costeiros (Junk 2002; Belém 2005). Além disso, Junk (2002) também aponta que os conceitos de planejamento ambiental e os níveis de conscientização e informação popular deficitários, junto à ineficiência dos orgãos gestores do meio ambiente, são os principais causadores da destruição dessas áreas. Espera-se que os resultados aqui apresentados possam servir de alerta aos órgãos ambientais. Pesquisa eficiente e gestão sustentável dos recursos nos parecem um modelo essencial sob o qual podem ser equacionados e resolvidos muitos dos problemas socioambientais na APA Rio Capivara.

\section{Agradecimentos}

Os autores agradecem aos seguintes especialistas pela colaboração na identificação e/ou confirmação de algumas espécies: L.P. de Queiroz (Fabaceae), E. de Melo (Polygonaceae), C. van den Berg (Orchidaceae), J.G. Jardim e E.B. Souza (Rubiaceae), J.F. Pastore (Polygalaceae), R.P. Oliveira (Poaceae), M.C. Machado (Cactaceae), R.M. de Castro (Moraceae) e A.K.A. Santos (Melastomataceae); à Adriana Moraes, Magdalena e Rafaela Galante, pela ajuda durante atividades de campo; à Synara Mattos Leal, pelo auxílio com os registros fotográficos; à Dra. Marina Siqueira de Castro, pelas facilidades oferecidas a DBOSC e MHSF durante o desenvolvimento de uma das etapas do trabalho de campo. Agradecemos ainda aos curadores dos herbários ALCB, HUEFS e HRB pela disponibilidade de suas coleções; ao

\section{REFERÊNCIAS}

Albuquerque, U.P. 1999a. La importancia de los estudios etnobiológicos para establecimiento de estrategias de manejo y conservación en las florestas tropicales. Biotemas 12:31-47.

Albuquerque, U.P. 1999b. Manejo tradicional de plantas em regiões neotropicais. Acta Botanica Brasilica 13:307-315.

Albuquerque, U.P. \& Andrade, L.H.C. 2002. Conhecimento botânico tradicional e conservação em uma área de caatinga no estado de Pernambuco, Nordeste do Brasil. Acta Botanica Brasilica 16:273-285.

Alves, F.M.; Souza, V.C. \& Moraes, P.L.R. 2011. Mezilaurus revolutifolia (Lauraceae), a new species from Brazilian Atlantic Forest. Kew Bulletin 66(4): 505-509.

APG III 2009. An update of the Angiosperm Phylogeny Group classification for the orders and families of flowering plants: APG III. Botanical Journal of the Linnean Society 161: 105-121.

Araujo, D.S.D. 1992. Vegetation types of sandy coastal plains of tropical Brazil: a first approximation. In: U. Seeliger (ed.), Coastal Plant Communities of Latin America. Academic Press, New York, p. 337-347.

Araujo, D.S.D. \& Henriques, R.B.P. 1984. Análise florística das restingas do estado do Rio de Janeiro. In: L.D. Lacerda, D.S.D. Araujo, R. Cerqueira \& B. Turcq (eds), Restingas: origem, estrutura e processos. CEUFF, Niterói, p. 159-193.

Assis, A.M.; Pereira, O.J. \& Thomaz, L.D. 2004a. Fitossociologia de uma floresta de restinga no Parque Estadual Paulo César Vinha, Setiba, município de Guarapari (ES). Revista Brasileira de Botânica 27(2): 349-361.

Assis, A.M.; Thomaz, L.D. \& Pereira, O.J. 2004b. Florística de um trecho de floresta de restinga no município de Guarapari, Espírito Santo, Brasil. Acta Botanica Brasilica 18(1):
191-201.

Assumpção, J. \& Nascimento, M.T. 2000. Estrutura e composição florística de quatro formações vegetais de restinga no complexo lagunar Grussaí/Iquipari, São João da Barra, RJ, Brasil. Acta Botanica Brasilica 14(3): 301-315.

Begossi, A. 1998. Extractive reserve in the Brazilian Amazon: an example to be followed in the Atlantic Forest? Ciência \& Cultura 50(1): 24-28.

Belém, L. 2005. Litoral Norte ameaçado: mudança no zoneamento das áreas de proteção permite o surgimento de empreendimentos em locais que deveriam ser preservados. Jornal A Tarde. Salvador, 13 dez. 2005, p. 8.

Britto, I.C. \& Noblick, L.R. 1984. A importância de preservar as dunas de Itapoã e Abaeté. In: L.D. Lacerda, D.S.D. Araujo, R. Cerqueira \& B. Turcq (eds), Restingas: origem, estrutura e processos. CEUFF, Niterói, p. 269-274.

Britto, I.C.; Queiroz, L.P.; Guedes, M.L.S.; Oliveira, N.C. \& Silva, L.B. 1993. Flora fanerogâmica das dunas e lagoas do Abaeté, Salvador, Bahia. Sitientibus série Ciências Biológicas 11:31-46.

César, O. \& Monteiro, R. 1995. Florística e fitossociologia de uma floresta de restinga em Picinguaba (Parque Estadual da Serra do Mar), município de Ubatuba - SP. Naturalia 20: 89-105.

Costa, J.A.S. \& Ramalho, R. 2001. Ecologia da polinização em ambiente de duna tropical (APA do Abaeté, Salvador, Bahia, Brasil). Sitientibus série Ciências Biológicas 1(2): 141-153.

Diegues, A.C.S. 2000. Etnoconservação: novos rumos para a conservação da natureza. Hucitec e NUPAUB/USP, São Paulo.

Fabris, L.C. \& César, O. 1996. Estudos florísticos em uma mata 
Albuquerque, U.P. 1999a. La importancia de los estudios etnobiológicos para establecimiento de estrategias de manejo y conservación en las florestas tropicales. Biotemas 12:31-47.

Albuquerque, U.P. 1999b. Manejo tradicional de plantas em regiões neotropicais. Acta Botanica Brasilica 13: 307-315.

Albuquerque, U.P. \& Andrade, L.H.C. 2002. Conhecimento botânico tradicional e conservação em uma área de caatinga no estado de Pernambuco, Nordeste do Brasil. Acta Botanica Brasilica 16(3): 273-285.

Alves, F.M.; Souza, V.C. \& Moraes, P.L.R. de. 2011. Mezilaurus revolutifolia (Lauraceae), a new species from Brazilian Atlantic Forest. Kew Bulletin 66(4): 505-509.

APG III 2009. An update of the Angiosperm Phylogeny Group classification for the orders and families of flowering plants: APG III. Botanical Journal of the Linnean Society 161: $105-121$.

Araujo, D.S.D. 1992. Vegetation types of sandy coastal plains of tropical Brazil: a first approximation. In: U. Seeliger (ed.), Coastal Plant Communities of Latin America. Academic Press, New York, p. 337-347.

Araujo, D.S.D. \& Henriques, R.B.P. 1984. Análise florística das restingas do estado do Rio de Janeiro. In: L.D. Lacerda, D.S.D. Araujo, R. Cerqueira \& B. Turcq (eds.), Restingas: origem, estrutura e processos. CEUFF, Niterói, p. 159-193.

Assis, A.M.; Pereira, O.J. \& Thomaz, L.D. 2004a. Fitossociologia de uma floresta de restinga no Parque Estadual Paulo César Vinha, Setiba, município de Guarapari (ES). Revista Brasileira de Botânica 27(2): 349-361.

Assis, A.M.; Thomaz, L.D. \& Pereira, O.J. 2004b. Florística de um trecho de floresta de restinga no município de Guarapari, Espírito Santo, Brasil. Acta Botanica Brasilica 18(1): 191-201.

Assumpção, J. \& Nascimento, M.T. 2000. Estrutura e composição florística de quatro formações vegetais de restinga no complexo lagunar Grussaí/Iquipari, São João da Barra, RJ, Brasil. Acta Botanica Brasilica 14(3): 301-315.

Begossi, A. 1998. Extractive reserve in the Brazilian Amazon: an example to be followed in the Atlantic Forest? Ciência \& Cultura 50(1): 24-28.

Belém, L. 2005. Litoral Norte ameaçado: mudança no zoneamento das áreas de proteção permite o surgimento de empreendimentos em locais que deveriam ser preservados. Jornal A Tarde. Salvador, 13 dez. 2005, p. 8.

Britto, I.C. \& Noblick, L.R. 1984. A importância de preservar as dunas de Itapoã e Abaeté. In: L.D. Lacerda, D.S.D. Araujo, R. Cerqueira \& B. Turcq (eds.), Restingas: origem, estrutura e processos. CEUFF, Niterói, p. 269-274.

Britto, I.C.; Queiroz, L.P.; Guedes, M.L.S.; Oliveira, N.C. \& Silva, L.B. 1993. Flora fanerogâmica das dunas e lagoas do Abaeté, Salvador, Bahia. Sitientibus série Ciências Biológicas 11:31-46.

César, O. \& Monteiro, R. 1995. Florística e fitossociologia de uma floresta de restinga em Picinguaba (Parque Estadual da Serra do Mar), município de Ubatuba - SP. Naturalia 20: 89-105.

Costa, J.A.S. \& Ramalho, R. 2001. Ecologia da polinização em ambiente de duna tropical (APA do Abaeté, Salvador, Bahia, Brasil). Sitientibus série Ciências Biológicas 1(2): 141-153.

Diegues, A.C.S. 2000. Etnoconservação: novos rumos para a conservação da natureza. Hucitec e NUPAUB/USP, São Paulo.

Fabris, L.C. \& César, O. 1996. Estudos florísticos em uma mata litorânea no sul do estado do Espírito Santo. Boletim do Museu de Biologia Mello Leitão 5: 15-46.

Fonseca-Kruel, V.S. \& Peixoto, A.L. 2004. Etnobotânica na Reserva Extrativista Marinha de Arraial do Cabo, RJ, Brasil. Acta Botanica Brasilica 18(1): 177-190.
Fraga, C.N. 2008. Three new species of Davilla (Dilleniaceae) from Bahia, Brazil. Brittonia 60(4) 355-361.

Freidenburg, L.K. 1997. Physical effects of habitat fragmentation. In: P.L. Fiedler \& P.M. Kareiva (eds), Conservation Biology for the Coming Decade. Chapman and Hall, New York, p. 66-79.

Freire, M.S.B. 1990. Levantamento florístico do Parque Estadual das Dunas de Natal. Acta Botanica Brasilica 4(2): 41-59.

Holmgren, P.K.; Holmgren, N.H. \& Barnett, L.C. 1990. Index Herbariorum. Part I: the herbaria of the world. Association of Plant Taxonomy and New York Botanical Garden, New York.

IBGE (Instituto Brasileiro de Geografia e Estatística) 2004. Flora das Restingas do Litoral Norte da Bahia: Costa dos Coqueiros e Salvador. Projeto Flora/Fauna - UE/BA Microbiological Diagnosis: text and color atlas. Editora Médica e Científica Ltda Herbário Radambrasil, Salvador.

Junk, W.J. 2002. Long-term environmental trends and the future of tropical wetlands. Environmental Conservation 29(4): 414-435.

Kemenes, A. 2003. Distribuição espacial da flora terrestre fanerogâmica do Parque Nacional Marinho de Abrolhos, BA. Revista Brasileira de Botânica 26(2): 141-150.

Kita, K.K. \& Souza, M.C. 2003. Levantamento florístico e fitofisionomia da lagoa Figueira e seu entorno, planície alagável do alto rio Paraná, Porto Rico, estado do Paraná, Brasil. Acta Scientiarum: Biological Sciences 25(1): 145-155.

Lemos, M.C., Pellens, R. \& Lemos, L.C. 2001. Perfil e florística de dois trechos de mata litorânea no município de Maricá-RJ. Acta Botanica Brasilica 15(3): 321-334.

Lennartsson, T. 2002. Extinction thresholds and disrupted plantpollinator interactions in fragmented plant populations. Ecology 83(11): 3060-3072.

Lundberg, S. \& Ingvarsson, P.K. 1998. Population dynamics of resource limited plants and their pollinators. Theoretical Population Biology 54: 44-49.

Mantovani, W. 1992. A vegetação sobre a restinga de Caraguatatuba, SP. Revista do Instituto Florestal de São Paulo 4: 139-144.

Marques, J.G.W. 1995. Pescando Pescadores: etnoecologia abrangente no Baixo São Francisco. NUPAUB/USP, São Paulo.

Meffe, G.K.; Carroll, C.R. \& Pimm, S.L. 1997. Community- and ecosystem-level conservation: species interactions, disturbance regimes, and invading species. In: G.K. Meffe \& C.R. Carroll (eds), Principles of Conservation Biology. Sinauer Associates, Sunderland, p. 235-267.

Melo, E. 1996. Levantamento das espécies de Coccoloba (Polygonaceae) da restinga do estado da Bahia, Brasil. Sitientibus série Ciências Biológicas 15: 49-59.

Melo, S.; Lacerda, V.D. \& Hanazaki, N. 2008. Espécies de restinga conhecidas pela comunidade do Pântano do Sul, Florianópolis, Santa Catarina, Brasil. Rodriguésia 59(4): 799-812.

Miranda, T.M. \& Hanazaki, N. 2008. Conhecimento e uso de recursos vegetais de restinga por comunidades das Ilhas do Cardoso (SP) e de Santa Catarina (SC), Brasil. Acta Botanica Brasilica 22(1): 203-215.

MMA (Ministério do Meio Ambiente) 2000. Avaliação e Ações Prioritárias para a Conservação da Biodiversidade da Mata Atlântica e Campos Sulinos. MMA/SBF, Brasília.

Mori S.A.; Boom, B.M. \& Prance, G.T. 1981. Distribution patterns and conservation of the Eastern Brazilian coastal forest tree species. Brittonia 33(2): 233-245.

Müller, S.C. \& Waechter, J.L. 2001. Estrutura sinusial dos componentes herbáceo e arbustivo de uma floresta costeira subtropical. Revista Brasileira de Botânica 24(4): 263-272. 
Pereira, M.C.A.; Araujo, D.S.D. \& Pereira, O.J. 2001. Estrutura de uma comunidade arbustiva da restinga de Barra de Maricá - RJ. Revista Brasileira de Botânica 24(3): 273-281.

Pereira, M.C.A.; Cordeiro, S.Z. \& Araujo, D.S.D. 2004. Estrutura do estrato herbáceo na formação aberta de Clusia do Parque Nacional da Restinga de Jurubatiba, RJ, Brasil. Acta Botanica Brasilica 18(3): 677-687.

Pereira, O.J. \& Araujo, D.S.D. 2000. Análise florística das restingas dos Estados do Espírito Santo e Rio de Janeiro. In: F.A. Esteves \& L.D. Lacerda (eds), Ecologia de Restingas e Lagoas Costeiras. NUPEM/UFRJ, Macaé, p. 25-63.

Pereira, O.J. \& Assis, A.M. 2000. Florística da restinga de Camburi. Acta Botanica Brasilica 14(1): 99-111.

Pinto, G.C.P.; Bautista, H.P. \& Ferreira, J.D.C.A. 1984a. A restinga do litoral nordeste do estado da Bahia. In: L.D. Lacerda, D.S.D. Araujo, R. Cerqueira \& B. Turcq. (eds), Restingas: origem, estrutura e processos. CEUFF, Niterói, p. 195-216.

Pinto, G.C.P.; Bautista, H.P. \& Ferreira, J.D.C.A. 1984b. $A$ Restinga do Litoral Nordeste do Estado da Bahia. Projeto RADAMBRASIL/Divisão de Vegetação, Salvador.

Popovkin, A.V.; Mathews, K.G.; Santos, J.C.M.; Molina, M.C. \& Struwe, L. 2011. Spigelia genuflexa (Loganiaceae), a new geocarpic species from the Atlantic forest of northeastern Bahia, Brazil. PhytoKeys 6: 47-65.

Posey, D.A. 1983. Indigenous knowledge and development: an ideological bridge to the future. Ciência e Cultura 35(7): 877-894.

Queiroz, E.P. 2007. Levantamento florístico e georreferenciamento das espécies com potencial econômico e ecológico em restinga de Mata de São João, Bahia, Brasil. Biotemas 20(4): 41-47.

Rizzini, C.T. 1997. Tratado de Fitogeografia do Brasil. Âmbito Cultural, Rio de Janeiro.

Scherer, A.; Maraschin-Silva, F. \& Baptista, L.R.M. 2005. Florística e estrutura do componente arbóreo de matas de restinga arenosa no Parque Estadual de Itapuã, RS, Brasil.
Acta Botanica Brasilica 19(4): 717-726.

SEI (Superintendência de Estudos Econômicos e Sociais do Estado da Bahia) 1998. Análise dos atributos climáticos do estado da Bahia. Série Estudos e Pesquisas 38, Salvador.

SEMARH/DUC \& CRA 2005. Boletim SEIA-APAs Estaduais. Disponível em http://www.sei.ba.gov.br/apa/apacapivara/template01.cfm?idCodigo=119; acesso em 19 nov. 2005 .

Silva J.G. \& Oliveira, A.S. 1989. A vegetação de restinga no município de Maricá, RJ. Acta Botanica Brasilica 3(2): 253-272.

Silva, S.M. 2005. Avaliação e Ações Prioritárias para a Conservação da Biodiversidade da Zona Costeira e Marinha: diagnóstico das restingas no Brasil. Disponível em http://www.bdt.fat.org.br/workshop/costa/restinga/; acesso em 19 nov. 2005.

Smith, A.R.; Pryer, K.M.; Schuettpelz, E.; Korall, P.; Schneider, H. \& Wolf, P.G. 2006. A classification for extant ferns. Taxon 55(3): 705-731.

Stehmann, J.R.; Forzza, R.C.; Salino, A.; Sobral, M.; Costa, D.P. \& Kamino, L.H.Y. 2009. Diversidade taxonômica na Floresta Atlântica. In: J.R. Stehmann, R.C. Forzza, A. Salino, M. Sobral, D.P. Costa \& L.H.Y Kamino (eds), Plantas da Floresta Atlântica. Jardim Botânico do Rio de Janeiro, Rio de Janeiro, p. 3-12.

Sugiyama, M. 1998. Estudo de florestas da restinga da Ilha do Cardoso, Cananéia, São Paulo. Boletim do Instituto de Botânica 11: 19-159.

Teixeira, R.L. 2001. Comunidade de lagartos da restinga de Guriri, São Mateus - ES, Sudeste do Brasil. Atlântica 23: 77-84.

Thomas, W.W.; Jardim, J.G.; Fiaschi. P. \& Amorim, A.M.A. 2003. Lista preliminar das angiospermas localmente endêmicas do sul da Bahia e norte do Espirito Santo, Brasil. In: P.I. Prado, E.C. Landau, R.T. Moura, L.P.S. Pinto, G.A.B. Fonseca \& K. Alger (eds), Corredor de Biodiversidade da Mata Atlântica do Sul da Bahia. CD-ROM. IESBConservation International, Ilhéus. 
Apêndice. Lista das espécies encontradas nas restingas de Arembepe, Camaçari, Bahia. Coletores: AG- A.M. Giulietti; AR- A. Rapini; DC- D. Cardoso; EB- E. Barbosa; EG- E. Gusmão; EQ- E.P. Queiroz; ES- E.B. Souza; GH- G. Hatschbach; GP- G.C.P. Pinto; HB- H.P. Bautista; LG- L. Gomes; LN- Larry Noblick; LP- L. Pacca; MA- M. Alves; NC- N. Carvalho; RK- R. Kuhn-Neto. Hábitos: arv-árvore; arb- arbusto; subarb- subarbusto; epi- epífita; erv- erva; para- hemi ou holoparasita; trep- trepadeira. * indica as espécies endêmicas das restingas de Arembepe e/ou áreas próximas do litoral norte da Bahia. " indica as espécies representadas no Material Suplementar (Figuras S1-S6).

\begin{tabular}{|c|c|c|c|}
\hline FAMÍLIA/Espécie & Testemunho & Hábito & Nome popular \\
\hline \multicolumn{4}{|c|}{ MONILÓFITAS } \\
\hline \multicolumn{4}{|l|}{ BLECHNACEAE } \\
\hline -Blechnum serrulatum Rich. & EQ 3886 & erv & feto-do-brejo \\
\hline \multicolumn{4}{|l|}{ DENNSTAEDTIACEAE } \\
\hline Pteridium aquilinum (L.) Kuhn & EQ 3891 & subarb & feto-das-taperas \\
\hline \multicolumn{4}{|l|}{ LINDSAEACEAE } \\
\hline Lindsaea sp. & EQ 1633 & erv & \\
\hline \multicolumn{4}{|l|}{ LYGODIACEAE } \\
\hline Lygodium volubile $\mathrm{Sw}$. & EQ 3882 & trep & cipó-balainho \\
\hline \multicolumn{4}{|l|}{ PTERIDACEAE } \\
\hline Acrostichum aureum L. & EQ 3889 & subarb & rabo-de-macaco \\
\hline Adiantum sp. & EQ 3894 & erv & \\
\hline \multicolumn{4}{|l|}{ THELYPTERIDACAE } \\
\hline Thelypteris interrupta (Willd.) K.Iwats. & EQ 1594 & erv & \\
\hline
\end{tabular}

\section{ANGIOSPERMAS}

Alternanthera maritima (Mart.) A.St.-Hil.

Blutaparon portulacoides (A.St.-Hil.) Mears

LP 11 erv

Gomphrena duriuscula Moq.

LP 4

DC 1069

erv

\section{ANACARDIACEAE}

-Anacardium occidentale L.

-Schinus terebinthifolius Raddi

DC 1055 arv

EQ 1563

DC 1130

arb, arv

cajueiro

-Tapirira guianensis Aubl.

EQ 3833

arv

aroeira

Thyrsodium spruceanum Benth.

arv

ANNONACEAE

Annona glabra L.

DC 1006

arb

araticum

APOCYNACEAE

*Blepharodon costae Fontella \& Morillo

AR 1072 trep

Ditassa crassifolia Decne.

EQ 1639

trep

-Hancornia speciosa Gomes

MA 861

-Mandevilla scabra (Hoffmanns. ex Roem. \& Schult.) K.Schum.

EQ 692

AQUIFOLIACEAE

Ilex theezans Mart.

EQ 1329

arb, arv

mangaba

ARACEAE

-Anthurium affine Schott

DC 1062

trep

alamanda

Philodendron imbe Mart.

EQ 1626

DC 1093

arb

caúna

Philodendron leal-costae Mayo \& G.M.Barroso

subarb

folha-de-urubu

ARALIACEAE

Hydrocotyle bonariensis Lam.

EQ $3807 \quad$ erv 
ARECACEAE

*"Allagoptera brevicalyx M.Moraes

\begin{tabular}{|c|c|c|}
\hline DC 1080 & subarb & caxulé \\
\hline EQ 2288 & arb & piaçava \\
\hline EQ 1241 & subarb & tucum \\
\hline DC 1090 & arb, arv & licurioba \\
\hline EQ 3808 & arb & cura-facada \\
\hline DC 1043 & arb & \\
\hline EQ 1579 & subarb & \\
\hline EQ 735 & erv & mal-me-quer \\
\hline EQ 3809 & subarb & \\
\hline EQ 3883 & subarb & \\
\hline DC 1138 & subarb & \\
\hline EQ 3816 & erv & \\
\hline EQ 737 & subarb & \\
\hline DC 1040 & erv & \\
\hline EQ 736 & subarb & \\
\hline EQ 3819 & subarb & \\
\hline EQ 1655 & trep & \\
\hline EQ 672 & subarb & balainho-de-velho \\
\hline EQ 1549 & erv & mal-me-quer \\
\hline EQ 3815 & erv & mal-me-quer \\
\hline 1405 & arv & ipê-rosa \\
\hline 1147 & arb, arv & caixeteira \\
\hline
\end{tabular}

-Attalea funifera Mart. ex Spreng.

*"Bactris soeiroana Noblick ex A.J.Hend.

-Syagrus schizophylla (Mart.) Glassman

\section{ASTERACEAE}

Acritopappus confertus (Gardner) R.M.King \& H.Rob.

Baccharis salzmannii DC.

Bahianthus viscosus (Spreng.) R.M.King \& H.Rob.

Calea angusta S.F.Blake

Conocliniopsis prasiifolia (DC.) R.M.King \& H.Rob.

Conyza sumatrensis (Retz.) E.Walker

Elephantopus hirtiflorus DC.

Emilia fosbergii Nicolson

Lepidaploa arenaria (Mart. ex DC.) H.Rob.

Lepidaploa cotoneaster (Willd. ex Spreng.) H.Rob.

Lepidaploa mucronifolia (DC.) H.Rob.

Litothamnus nitidus (DC.) W.C.Holmes

Mikania sp.

Platypodanthera melissifolia (DC.) R.M.King \& H.Rob.

Sphagneticola trilobata (L.) Pruski

DC 1147

Wedelia alagoensis Baker

BIGNONIACEAE

Handroanthus impetiginosus (Mart. ex DC.) Mattos

Tabebuia elliptica (DC.) Sandwith

BONNETIACEAE

-Bonnetia stricta (Nees) Nees \& Mart.

EQ 1630

arv

mangue-sereno

BORAGINACEAE

Cordia curassavica (Jacq.) Roem. \& Schult.

Euploca polyphylla (Lehm.) J.I.M.Melo \& Semir

EQ 3811 subarb

Varronia multispicata (Cham.) Borhidi

LG 1 erv

\section{BROMELIACEAE}

-Aechmea marauensis Leme

EQ 745

arb

Aechmea multiflora L.B.Sm.

EQ 1224 erv

*"Hohenbergia littoralis L.B.Sm.

EQ 3810

erv

DC 1018

erv

chupa-chupa

BURMANNIACEAE

Burmannia flava Mart.

EQ 1147 erv

Burmannia capitata Mart.

EQ 1621

erv

\section{BURSERACEAE}

*"Protium bahianum Daly

DC 1024 arb

Protium heptaphyllum (Aubl.) Marchand

EQ 711

arb

arv

amescla-açu

CACTACEAE

Cereus fernambucensis Lem.

DC $1101 \quad$ arb

-Melocactus violaceus subsp. margaritaceus N.P.Taylor

DC $1081 \quad$ erv

gravatá 
Pilosocereus catingicola subsp. salvadorensis (Werderm.) Zappi DC 1082 arv

CALOPHYLLACEAE

Calophyllum brasiliense Cambess.

*"Kielmeyera argentea Choisy

CELASTRACEAE

Maytenus distichophylla Mart. ex Reissek

\section{CHRYSOBALANACEAE}

Chrysobalanus icaco L.

Hirtella ciliata Mart. \& Zucc.

-Hirtella racemosa Lam.

CLEOMACEAE

Dactylaena microphylla Eichler

\section{CLUSIACEAE}

Clusia nemorosa G.Mey

Symphonia globulifera L.f.

\section{COMBRETACEAE}

-Conocarpus erectus L.

\section{COMMELINACEAE}

Commelina elegans Kunth

CONVOLVULACEAE

Evolvulus cordatus Moric.

Evolvulus jacobinus Moric.

Ipomoea asarifolia Roem. \& Schult.

Ipomoea bahiensis Willd. ex Roem. \& Schult.

Ipomoea imperati (Vahl) Griseb.

Ipomoea pes-caprae (L.) R.Br.

\section{COSTACEAE}

Costus spiralis Roscoe

CYPERACEAE

Abildgaardia baeothryon A.St.-Hil.

Bulbostylis junciformis (Kunth) C.B.Clarke

Bulbostylis sp.

Cyperus aggregatus (Willd.) Endl.

Cyperus distans G.Mey

Cyperus haspan L.

Cyperus laxus Lam.

Cyperus maritimus Poir.

Cyperus odoratus L.

Cyperus rotundus $\mathrm{L}$.

Cyperus subcastaneus D.A.Simpson

Eleocharis interstincta (Vahl) Roem. \& Schult.

Eleocharis geniculata (L.) Roem. \& Schult.

Eleocharis sp.

Fimbristylis cymosa R.Br.

Fuirena umbellata Rottb.

Lagenocarpus rigidus Nees

Rhynchospora barbata (Vahl) Kunth

-Rhynchospora cephalotes (L.) Vahl

Rhynchospora exaltata Kunth

$\begin{array}{lll}\text { EQ } 3812 & \text { arv } & \text { alandim } \\ \text { DC } 1027 & \text { arb } & \end{array}$

DC 994 arb, arv pau-de-açúcar

DC 1146 arb guajiru, abageru

EQ 3820 arb, arv murtinha

RK $10 \quad$ arb

DC 1099 erv

$\begin{array}{lll}\text { EQ } 3817 & \text { arv } & \text { ceboleira } \\ \text { EQ } 3814 & \text { arv } & \text { landim }\end{array}$

DC 990 arb, arv mangue-de-bolota

EQ 720 erv marianinha

$\begin{array}{lll}\text { EQ 1604 } & \text { erv } & \\ \text { EQ 3813 } & \text { erv } & \\ \text { EQ 694 } & \text { erv } & \\ \text { EQ 673 } & \text { trep } & \text { jitirana-de-leira } \\ \text { EQ 3834 } & \text { erv } & \text { salsa } \\ \text { EQ 1561 } & \text { erv } & \text { salsa-da-praia }\end{array}$

EQ 626 subarb bastão-do-imperador

GP 291-83 erv

EQ 3825 erv

EQ 1233 erv

EQ 3821 erv

EQ 3827 erv

junca

DC 1030 erv

EQ 3829 erv

EQ 1573 erv

EQ 1592 erv

EQ 3826 erv

HB 805 erv

EQ 3822 erv

MA 876 erv

EQ 1161 erv

EQ 3823 erv

DC 1029 erv

DC 1064 erv tiririca

DC 1031 erv

DC 1131 erv

DC 1028 erv 
Rhynchospora holoschoenoides (Rich.) Herter

Rhynchospora riparia (Nees) Boeckeler

Scleria bracteata Cav.

\section{DILLENIACEAE}

- Curatella americana L.

- Davilla flexuosa A.St.-Hil.

-Doliocarpus sellowianus Eichler

Tetracera breyniana Schltdl.

\section{DIOSCOREACEAE}

- Dioscorea ovata Vell.

\section{DROSERACEAE}

Drosera intermedia Hayne

\section{EBENACEAE}

Diospyros duartei Cavalcante

\section{ERICACEAE}

Gaylussacia brasiliensis (Spreng.) Meisn.

\section{ERIOCAULACEAE}

Actinocephalus ramosus (Wikstr.) Sano

- Comanthera imbricata (Körn.) L.R.Parra \& Giul.

Leiothrix flavescens (Bong.) Ruhland

Leiothrix restingensis (Moldenke) Giul.

Leiothrix sp.

Paepalanthus klotzschianus Körn.

Paepalanthus sp.

Syngonanthus longipes Gleason

\section{ERYTHROXYLACEAE}

Erythroxylum passerinum Mart.

*"Erythroxylum leal-costae Plowman

\section{EUPHORBIACEAE}

Chamaesyce hyssopifolia (L.) Small

Cnidoscolus urens (L.) Arthur

Croton glandulosus L.

Croton sellowii Baill.

Euphorbia gymnoclada Boiss.

Maprounea brasiliensis A.St.-Hil.

- Pera glabrata (Schott) Poepp. ex Baill.

Sebastiania corniculata (Vahl) Müll.Arg.

Sebastiania sp.

\section{FABACEAE-Caesalpinioideae}

Chamaecrista cytisoides (DC. ex Collad.) H.S.Irwin \& Barneby

Chamaecrista duartei (H.S.Irwin) H.S.Irwin \& Barneby

Chamaecrista flexuosa (L.) Greene

Chamaecrista ramosa (Vogel) H.S.Irwin \& Barneby

Chamaecrista rotundifolia (Pers.) Greene

Phanera outimouta (Aubl.) L.P.Queiroz

Senna macranthera (DC. ex Collad.) H.S.Irwin \& Barneby

FABACEAE-Faboideae

-Abrus precatorius L.

Andira nitida Mart. ex Benth.

$\begin{array}{ll}\text { MA } 877 & \text { erv } \\ \text { MA } 875 & \text { erv } \\ \text { EQ 1406 } & \text { erv }\end{array}$

DC 1110

DC 1036

arb, arv

tiririca-de-navalha

EQ 3824

EQ 1629

EQ 3831

EQ 975

EQ 3839

DC 1042

HB 814

EQ 677

DC 1087

AG 2451

DC 1070

HB 818

DC 1048

EQ 1612

EQ 1330

EQ 3828

EQ 1572

EQ 3832

EQ 1588

DC 1053

EQ 3840

EQ 3835

DC 1058

DC 1137

EQ 1583

EQ 1556

EQ 1177

EQ 3837

DC 1017

EQ 754b

EQ 3836

EQ 625

EQ 3838

DC 1094 trep

trep

trep

trep

cará

erv

arb

cabeleira

arb

subarb

erv

erv

erv

erv

erv

erv

erv

arb

estralador

arb

erv

arb

subarb

subarb

erv

arb

arv

subarb

erv

subarb

sucupirinha subarb subarb subarb subarb

arb

pata-de-vaca

arb

trep

arv pau-de-tamanco

erva-de-santa-luzia cansancão

velame cabeça-de-saúva 
-Bowdichia virgilioides Kunth

Calopogonium mucunoides Desv.

Canavalia dictyota Piper

Canavalia rosea (Sw.) DC.

-Centrosema brasilianum (L.) Benth.

Centrosema coriaceum Benth.

Clitoria guianensis (Aubl.) Benth.

Clitoria laurifolia Poir.

Crotalaria lanceolata E.Mey

Crotalaria pallida Aiton

Crotalaria retusa L.

Dalbergia ecastaphyllum (L.) Taub.

Desmodium adscendens (Sw.) DC.

Desmodium barbatum (L.) Benth.

-Dioclea lasiophylla Mart. ex Benth.

Dioclea violacea Mart. ex Benth.

Indigofera microcarpa Desv.

Indigofera suffruticosa Mill.

Macroptilium lathyroides (L.) Urb.

-Leptolobium bijugum Vogel

Rhynchosia minima (L.) DC.

-Sophora tomentosa L.

Stylosanthes gracilis Mart.

Stylosanthes guianensis (Aubl.) Sw.

Stylosanthes scabra Vog.

Stylosanthes viscosa (L.) Sw.

-Swartzia apetala Raddi

Swartzia polita (R.S.Cowan) Torke

Vigna halophila (Piper) Maréchal, Mascherpa \& Stainier

Vigna luteola (Jacq.) Benth.

Zornia diphylla (L.) Pers.

Zornia glabra Desv.

\section{FABACEAE-Mimosoideae}

Abarema filamentosa (Benth.) Pittier

Inga capitata Desv.

Inga laurina (Sw.) Willd.

Mimosa pigra L.

Mimosa pudica $\mathrm{L}$.

Mimosa somnians Humb. \& Bonpl. ex Willd.

"Neptunia plena (L.) Benth.

\section{GENTIANACEAE}

Schultesia doniana Progel

Schultesia guianensis (Aubl.) Malme

HELICONIACEAE

Heliconia psittacorum L.f.

HUMIRIACEAE

-Humiria balsamifera Aubl.

HYDROPHYLLACEAE

Hydrolea spinosa $\mathrm{L}$.

\begin{tabular}{|c|c|c|}
\hline EQ 3845 & $\operatorname{arv}$ & sucupira \\
\hline EQ 1584 & trep & \\
\hline EQ 3842 & trep & \\
\hline EB 3 & erv & feijão-da-praia \\
\hline DC 1109 & trep & xibiu-de-nega \\
\hline EQ 708 & trep & \\
\hline EQ 629 & trep & \\
\hline DC 1120 & subarb & \\
\hline EQ 3897 & arb & \\
\hline EQ 664 & subarb & cascaveleira \\
\hline DC 1111 & subarb & guizo-de-cascavel \\
\hline DC 991 & arb & bugi \\
\hline EQ 3848 & subarb & carrapicho-beiço-de-boi \\
\hline DC 1103 & erv & barbadinho \\
\hline EQ 706 & trep & \\
\hline EQ 3846 & trep & mucunã \\
\hline EQ 751a & erv & \\
\hline EQ 707 & arb & anil \\
\hline DC 1001 & trep & \\
\hline EQ 3841 & arb, arv & angico-de-litoral \\
\hline EQ 642 & trep & matineta \\
\hline EQ 703 & arb & feijão-da-costa \\
\hline EQ 724 & subarb & \\
\hline EQ 1574 & subarb & vassourinha \\
\hline EQ 637 & subarb & \\
\hline DC 1102 & subarb & melosa \\
\hline DC 1065 & arb & fruto-de-araquã \\
\hline EQ 3843 & arb & pau-sangue \\
\hline EQ 710 & trep & \\
\hline DC 1000 & trep & \\
\hline EQ 635 & erv & \\
\hline DC 1106 & erv & \\
\hline EQ 3844 & arb, arv & barbatimão \\
\hline DC 1089 & arv & ingá \\
\hline EQ 1399 & arv & ingá-de-macaco \\
\hline DC 1112 & arb & \\
\hline EQ 3849 & arb & malícia-de-mulher \\
\hline DC 1139 & arb & \\
\hline DC 999 & arb & \\
\hline EQ 1230 & erv & \\
\hline DC 1015 & erv & \\
\hline EQ 3850 & erv & bananeirinha-do-mato \\
\hline DC 1060 & arb & umiri-de-cheiro \\
\hline DC 1005 & subarb & \\
\hline
\end{tabular}


HYPERICACEAE

-Vismia guianensis (Aubl.) Choisy

$\begin{array}{ll}\text { DC 1132 } & \text { arb } \\ \text { DC 1092 } & \text { arv } \\ \text { EQ 1141 } & \text { erv } \\ & \\ \text { DC 1022 } & \text { erv, subarb } \\ \text { GH 63079 } & \begin{array}{l}\text { subarb } \\ \text { EQ 3851 }\end{array} \\ & \text { erv, subarb } \\ \text { EQ 3856 } & \text { arb } \\ \text { EQ 949 } & \text { arb } \\ \text { EQ 3853 } & \text { subarb } \\ \text { EQ 3855 } & \text { arb }\end{array}$

ICACINACEAE

Emmotum affine Miers

IRIDACEAE

-Neomarica sabinii (Lindley) Chukr.

KRAMERIACEAE

Krameria bahiana B.B.Simpson

Krameria grandiflora A.St.-Hil.

Krameria tomentosa A.St.-Hil.

LAMIACEAE

Eriope hypenioides Mart.ex Benth.

Hyptis fruticosa Salzm. ex Benth.

Ocimum campechianum Mill.

-Vitex cymosa Bert. ex Spreng.

LAURACEAE

Cassytha filiformis L.

EQ 1590

para

Ocotea notata (Nees \& Mart.) Mez

DC 1073

arb, arv

capianga

\section{LECYTHIDACEAE}

-Eschweilera ovata (Cambess.) Miers

LN 2465 arv biriba

\section{LENTIBULARIACEAE}

Utricularia gibba $\mathrm{L}$.

Utricularia subulata $\mathrm{L}$.

QQ 3859 erv

Utricularia sp.

EQ 3854 erv

\section{LOGANIACEAE}

Spigelia anthelmia $\mathrm{L}$.

EQ 3852 erv

\section{LORANTHACEAE}

- Psittacanthus dichroos Mart.

Psittacanthus robustus Mart.

Struthanthus polyrhizus Mart.

Struthanthus syringifolius Mart.

LYTHRACEAE

Cuphea brachiata Koehne

- Cuphea flava Spreng.

Cuphea sessilifolia Mart.

\section{MALPIGHIACEAE}

Barnebya harleyi W.R.Anderson \& B.Gates

-Byrsonima bahiana W.R.Anderson

- Byrsonima coccolobifolia Kunth

EQ 979 erv

$\begin{array}{lll}\text { DC } 1007 & \text { para } & \text { erva-de-passarinho } \\ \text { DC } 997 & \text { para } & \text { erva-de-passarinho } \\ \text { DC } 1123 & \text { para } & \text { erva-de-passarinho } \\ \text { HB } 806 & \text { para } & \end{array}$

Byrsonima dealbata Griseb.

*"Byrsonima microphylla A.Juss.

-Byrsonima sericea DC.

DC 1050

subarb

sete-sangrias

EQ 683 subarb

EQ 3830 subarb

Stigmaphyllon ciliatum (Lam.) A.Juss.

-Stigmaphyllon paralias A.Juss.

\section{MALVACEAE}

Pavonia cancellata (L.) Cav.

Sida linifolia Cav.

Sida cordifolia L.

Triumfetta semitriloba Jacq.

EQ 1417 arb

EQ 1619 arb

EQ 3859 arb

EQ 1613 arb

GP 306-83 arb

EQ 1564 arb,

DC 1150 trep

DC 1180 subarb

$\begin{array}{lll}\text { DC } 1107 & \text { erv } & \text { poca-poca } \\ \text { EQ } 3861 & \text { subarb } & \\ \text { EQ } 671 & \text { subarb } & \\ \text { EQ } 3865 & \text { subarb } & \end{array}$


Urena lobata L.

Waltheria americana L.

-Waltheria cinerascens A.St.-Hil.

\section{MARANTHACEAE}

Stromanthe sp.

\section{MELASTOMATACEAE}

Acisanthera bivalvis Cogn.

Comolia ovalifolia Triana

Clidemia hirta (L.) D.Don

Marcetia ericoides Cogn.

Marcetia taxifolia (A.St.-Hil.) DC.

- Miconia ciliata DC.

Pterolepis cataphracta (Cham.) Triana

Pterolepis perpusilla (Naudin) Cogn.

Pterolepis polygonoides (DC.) Triana

Tibouchina elegans Cogn.

Tibouchina urceolaris Cogn.

\section{MOLLUGINACEAE}

Mollugo verticillata L.

\section{MORACEAE}

Ficus bahiensis C.C.Berg \& Carauta

Ficus nymphaeifolia Mill.

Ficus obtusiuscula (Miq.) Miq.

\section{MYRSINACEAE}

Myrsine parvifolia A.DC.

\section{MYRTACEAE}

- Campomanesia dichotoma (O.Berg) Mattos

Eugenia candolleana DC.

Eugenia hirta O.Berg

Marlierea neuwiedeana (O.Berg) Nied.

Myrcia bergiana O.Berg

Myrcia decorticans DC.

Myrcia splendens (Sw.) DC.

Myrcia salzmannii O.Berg

Myrcia tomentosa (Aubl.) DC.

Myrciaria floribunda (H.West ex Willd.) O.Berg

-Psidium guineense Sw.

\section{NYCTAGINACEAE}

"Guapira pernambucensis (Casar.) Lundell

\section{OCHNACEAE}

Ouratea suaveolens Engl.

Sauvagesia erecta L.

Sauvagesia sprengelii A.St.-Hil.

\section{ONAGRACEAE}

Ludwigia erecta (L.) H.Hara

Ludwigia octovalvis (Jacq.) P.H.Raven

\section{ORCHIDACEAE}

Brassavola tuberculata Hook.

Catasetum roseo-album (Hook.) Lindl.

$\begin{array}{lll}\text { EQ } 1600 & \text { erv } & \\ \text { EQ } 1610 & \text { erv } & \text { canela-de-juriti } \\ \text { DC } 1075 & \text { arb } & \end{array}$

EQ 3862 erv

$\begin{array}{lll}\text { EQ 655 } & \text { erv } & \\ \text { DC 1128 } & \text { erv, subarb } & \text { alecrim-da-praia } \\ \text { EQ } 1603 & \text { subarb } & \\ \text { DC 1044 } & \text { subarb } \\ \text { EQ 713 } & \text { erv } \\ \text { DC 1038 } & \text { arb } \\ \text { EQ 732 } & \text { subarb } \\ \text { EQ 714 } & \text { subarb } \\ \text { DC 1153 } & \text { subarb } \\ \text { DC 1045 } & \text { subarb } \\ \text { EQ 691 } & \text { arb } & \end{array}$

EQ 3866

$\begin{array}{ll}\text { DC 1056 } & \text { arv } \\ \text { EQ 742 } & \text { arv } \\ \text { EQ 738 } & \text { arv }\end{array}$

EQ $675 \quad$ arb, arv pororoca

$\begin{array}{ll}\text { EQ 3860 } & \text { arb } \\ \text { EQ } 1624 & \text { arb } \\ \text { EQ } 3857 & \text { arb } \\ \text { EQ } 680 & \text { arb } \\ \text { EQ } 3868 & \text { arb } \\ \text { EQ } 3871 & \text { arb } \\ \text { EQ 733 } & \text { arb } \\ \text { EQ } 648 & \text { arb } \\ \text { EQ } 3875 & \text { arb } \\ \text { DC } 1076 & \text { arb } \\ \text { EQ } 3873 & \text { arb }\end{array}$

DC $1011 \quad$ arb

DC 1091 arb

EQ 1616 erv, subarb

DC 1129 subarb

EQ 653 erv

DC 1002 erv

EQ $3874 \quad$ epi

DC 1041 erv 
- Cyrtopodium holstii L.C.Menezes

Encyclia dichroma (Lindl.) Schltr.

Encyclia oncidioides (Lindl.) Schltr.

-Epidendrum cinnabarinum Salzm. ex Lindl.

Epidendrum orchidiflorum Salzm. ex Lindl.

-Epistephium lucidum Cogn.

Habenaria pratensis (Lindl.) Rchb.f.

* Koellensteinia abaetana L.P.Queiroz

* Vanilla bahiana Hoehne

\section{OROBANCHACEAE}

Esterhazya caesarea (Cham. \& Schltdl.) V.C.Souza

PASSIFLORACEAE

- Passiflora edulis Sims

Passiflora foetida L.

Passiflora galbana Mast.

Passiflora mucronata Lam.

PENTAPHYLACACEAE

Ternstroemia brasiliensis Cambess.

PHYLLANTHACEAE

-Phyllanthus klotzschianus Müll.Arg.

PHYTOLACCACEAE

Phytolacca thyrsiflora Fenzl ex J.A.Schmidt

\section{PLANTAGINACEAE}

Achetaria scutellarioides Wettst.

Angelonia cornigera Hook.

Bacopa depressa (Benth.) Loefgr. \& Edwall

Bacopa gratioloides (Cham.) Edwall

POACEAE

Andropogon bicornis Forssk.

Andropogon leucostachyus Kunth

Andropogon selloanus (Hack.) Hack.

Aristida sp.

Axonopus aureus P.Beauv.

Chloris sp.

Panicum adspersum Trin.

Paspalum conspersum Schrad.

Paspalum cyanescens Nees

Paspalum divergens Doell

Paspalum cf. tenellum Willd.

Paspalum sp.

Schizachyrium condensatum Nees

Sporobolus indicus (L.) R.Br.

Trachypogon macroglossus Trin.

\section{POLYGALACEAE}

Polygala apressa Benth.

Polygala leptocaulis Cor. \& Gr.

Polygala martiana A.W.Benn.

Polygala monninoides Kunth

Polygala trichosperma L.

\begin{tabular}{|c|c|}
\hline DC 1108 & erv \\
\hline NC 1 & erv \\
\hline EQ 3870 & erv \\
\hline DC 1068 & subarb \\
\hline DC 1143 & erv \\
\hline DC 1085 & erv \\
\hline EQ 1642 & erv \\
\hline DC 1119 & erv \\
\hline EQ 3869 & trep \\
\hline
\end{tabular}

EQ 951 subarb

$\begin{array}{ll}\text { EQ 661 } & \text { trep } \\ \text { EQ 699 } & \text { trep } \\ \text { EQ 662 } & \text { trep } \\ \text { EQ 695 } & \text { trep }\end{array}$

EQ $3887 \quad$ arb

DC 1135 erv, subarb

EQ 1580 erv

EQ 1607 erv

EQ 739 erv

EQ 3885 erv

DC 1004 erv

EQ 623 erv

EQ 679 erv

EQ 1609 erv

RK 6 erv

EQ 1622 erv

EQ 3877 erv

EQ 1263 erv

EQ 627 erv

EQ 983 erv

EQ 964 erv

DC 1134 erv

EQ 1605 erv

EQ 1299 erv

EQ 1240 erv

DC 1023 erv

$\begin{array}{ll}\text { EQ } 966 & \text { erv } \\ \text { DC } 1008 & \text { erv } \\ \text { EQ } 660 & \text { erv } \\ \text { EQ } 1608 & \text { erv } \\ \text { DC } 1098 & \text { erv }\end{array}$

Sitientibus série Ciências Biológicas 12(1): 119-141. 2012. 
Polygala violacea Aubl.

POLYGONACEAE

Coccoloba alnifolia Casar.

- Coccoloba laevis Casar.

- Coccoloba ramosissima Wedd.

PORTULACACEAE

Portulaca sp.

RUBIACEAE

Alibertia edulis (Rich.) A.Rich. ex DC.

Amaioua guianensis Aubl.

Borreria capitata DC.

Borreria cymosa Cham. \& Schll.

Borreria laevis (Lam.) Griseb.

Borreria verticillata (L.) G.Mey.

Chiococca alba (L.) Hitchc.

-Chiococca brachiata Ruiz \& Pav.

Chomelia sp.

-Denscantia monodon (K.Schum.) E.L.Cabral \& Bacigalupo

Diodella apiculata (Willd. ex Roem. \& Schult.) Bacigalupo \& E.L.Cabral

Diodia radula (Willd. \& Hoffmanns. ex Roem. \& Schult.) Cham. \& Schltdl.

Diodia teres Walter

Guettarda platypoda DC.

Mitracarpus anthospermoides K.Schum.

Mitracarpus frigidus (Roem \& Schult.) K.Schum.

Mitracarpus sellowianus Cham. \& Schltdl.

Pagamea guianensis Aubl.

Perama hirsuta Aubl.

Richardia grandiflora (Cham. \& Schtdl.) Steud.

Sabicea grisea Cham. \& Schltdl.

Salzmannia nitida DC.

Staelia galioides DC.

Tocoyena bullata (Vell.) Mart.

\section{RUTACEAE}

Ertela trifolia (L.) Kuntze

- Esenbeckia grandiflora subsp. brevipetiolata Kaastra

SAPINDACEAE

Matayba guianensis Aubl.

Paullinia racemosa Wawra

Serjania corrugata Radlk.

\section{SAPOTACEAE}

Manilkara decrescens T.D.Penn.

- Manilkara salzmannii (A.DC.) H.J.Lam.

Manilkara subsericea (Mart.) Dubard.

-Pouteria grandiflora (A.DC.) Baehni

\section{SCHOEPFIACEAE}

Schoepfia brasiliensis A.DC.

\section{SIMAROUBACEAE}

Simaba cedron Planch.
DC 1105 erv

$\begin{array}{lll}\text { EQ } 3878 & \text { arb } & \text { cocozinho } \\ \text { DC } 1012 & \text { arb } & \text { cocó } \\ \text { DC } 1034 & \text { arb } & \end{array}$

EQ 690 erv

EQ $3880 \quad$ arb

DC 1077 arb

DC 1113 erv

EG 335 erv

EQ 686 erv, subarb corredeira

EQ 688 erv, subarb carqueja

DC 1144 arb

EQ 752b arb

EQ $3881 \quad$ arb

DC 1019 erv

EQ 3879 ery

EQ $741 \quad$ erv

HB 821 arb

ES 1335 erv

DC 1100 erv

EQ 3876 erv

HB $807 \quad$ arb

DC 1032 erv

DC 1115 subarb

ES 1336 erv

GP 299-83 arb

DC 1116 erv

EG 335 trep

DC 992 arb

EQ 3883 erv

DC 1136 arb mucambo

EQ 1239 arb, arv camboatã

EQ 749 trep

EQ 749 trep

DC 1025 arb

EQ 632 arb

EQ 1152 arv

EQ $3892 \quad$ arb

massaranduba-da-praia massaranduba bapeba

EQ $3872 \quad$ arb

EQ 3888 arb 
-Simaba cuneata A.St.-Hil. \& Tul.

SMILACACEAE

Smilax rufescens Griseb.

SOLANACEAE

Physalis angulata L.

Schwenckia americana L.

Solanum auriculatum Aiton

Solanum paniculatum L.

Solanum thomasiifolium Sendtn.

SURIANACEAE

-Suriana maritima L.

URTICACEAE

Cecropia glaziovii Snethl.

Cecropia pachystachya Trécul

VELLOZIACEAE

"Vellozia dasypus Seub.

VERBENACEAE

Lantana camara L.

Lippia harleyi Moldenke

Stachytarpheta canescens Kunth

Stachytarpheta sanguinea Mart. ex Schauer

Stachytarpheta maximiliani Schauer

VIOLACEAE

Hybanthus calceolaria (L.) Oken

\section{VOCHYSIACEAE}

Vochysia lucida C.Presl

XYRIDACEAE

Xyris jupicai Rich.

Xyris laxifolia Mart.

Xyris strobilifera Kunth
EQ $3893 \quad$ arb

DC 1052 trep uva-da-praia

$\begin{array}{lll}\text { EQ } 1599 & \text { subarb } & \text { canapu } \\ \text { DC } 1016 & \text { erv } & \\ \text { EQ } 1617 & \text { arb } & \text { caiçara } \\ \text { EQ } 3895 & \text { arb } & \text { jurubeba } \\ \text { EQ } 1557 & \text { arb } & \end{array}$

EQ $718 \quad$ arb

$\begin{array}{lll}\text { EQ } 3863 & \text { arv } & \text { embaúba-vermelha } \\ \text { EQ } 3896 & \text { arv } & \text { embaúba-branca }\end{array}$

DC 1035 subarb canela-de-ema

EQ 698 arb camará

GP 296-83 subarb gerebão

EQ 731 arb

EQ 3884 subarb gerebão

DC 1104 subarb

EQ 700 erv pecacunha

DC 1141 arv musserengue

$\begin{array}{ll}\text { EQ 646 } & \text { erv } \\ \text { DC 1009 } & \text { erv } \\ \text { EQ 3890 } & \text { erv }\end{array}$


